\title{
Flexibilität im Umgang mit mathematischen Situationsstrukturen - Eine Vorstudie für die Entwicklung eines Förderkonzepts zum Lösen additiver Textaufgaben
}

\author{
Laura Gabler (iD) Stefan Ufer
}

Eingegangen: 7. Januar 2019 / Angenommen: 11. Mai 2020 / Online publiziert: 27. Mai 2020

(C) Der/die Autor(en) 2020

Zusammenfassung Sprachliche Kompetenzen haben einen deutlichen Einfluss auf den mathematischen Kompetenzerwerb. Als Wirkmechanismus wird unter anderem auch die kognitive Nutzung von Sprache für die mathematische Wissenskonstruktion diskutiert. Vor allem bei Textaufgaben wird als wesentliches Schwierigkeitsmerkmal die sprachliche Umsetzung mathematischer Strukturen im Aufgabentext genannt. Frühere Studien zeigen, dass additive Textaufgaben, denen eine Vergleichssituation zugrunde liegt, eine besondere Herausforderung für Lernende darstellen. Um den Einfluss der sprachlichen Komplexität bei der Bearbeitung von Vergleichsaufgaben zu verringern, wurde in der Literatur bereits die Umdeutung in leichter zugängliche Situationsstrukturen als eine mögliche Bearbeitungsstrategie vorgeschlagen. Dies könnte Basis für ein Förderkonzept sein, welches Lernenden Strategien zur Umdeutung schwierigerer Textaufgaben in leichtere (z. B. Veränderungs- oder Ausgleichsaufgaben) vermittelt. In einer Vorstudie für die Entwicklung eines solchen Förderkonzepts wurde untersucht, ob Lernende Textaufgaben-Paare mit gleicher mathematischer Struktur, jedoch unterschiedlichen Situationsstrukturen identifizieren und dies zur Lösung von Vergleichsaufgaben nutzen. Umgesetzt wurde dies in einer Querschnittsstudie mit Paper-Pencil-Tests in acht Klassen der Jahrgangsstufe 2 $(N=139)$. Ein Ziel der Studie war es, bereits vorliegende Ergebnisse zur Schwierigkeit von Textaufgaben zu replizieren und zu systematisieren. Weiterhin war von Interesse, ob das vorherige Lösen einer Veränderungs- bzw. Ausgleichsaufgabe das darauffolgende Lösen einer als Vergleichssituation formulierten Aufgabe mit derselben mathematischen Struktur erleichtert. Die Ergebnisse deuten darauf hin, dass

L. Gabler $(\bowtie) \cdot$ S. Ufer

Mathematisches Institut, Ludwig-Maximilians-Universität München,

Theresienstraße 39, 80333 München, Deutschland

E-Mail: gabler@math.lmu.de

S. Ufer

E-Mail: ufer@math.lmu.de 
Lernende ohne explizite Instruktion unterschiedliche Beschreibungen derselben mathematischen Struktur nicht zum Lösen von Textaufgaben nutzen und werfen damit die Frage auf, inwiefern explizites Training in der Umdeutung von additiven Situationen die Lernenden bei der Bearbeitung derartiger Textaufgaben unterstützt.

Schlüsselwörter Flexibilität · semantische Struktur · additive Textaufgaben · Mengenvergleich

\title{
Flexibility when Dealing with Situational Structures in Mathematical Contexts-A Preliminary Study Investigating a Learning Framework on Solving Additive Word Problems
}

\begin{abstract}
Language competence has clear impact on the acquisition of mathematical skills. According to current discussions, one of the effect mechanisms could be the cognitive use of language for the construction of mathematical skills. In the context of word problems, the way mathematical structures are represented in the problem text verbally, is named as one of the crucial features of difficulty. Preceding studies show that additive word problems dealing with the comparison of sets are particularly challenging for mathematics learners. In order to minimize the influence of linguistic complexity features, research already proposed to teach strategies, which help to reinterpret word problems into easier accessible situational structures. This idea could be the foundation for a training program, which imparts strategies to reinterpret more difficult word problems into easier ones (e.g. change or equalizing tasks). A preliminary study aiming at the development of this sort of program was conducted to investigate, whether students identify pairs of word problems, which contain the same mathematical, but different situational structures, and use this information to solve comparison problems. This cross-sectional study was implemented with second graders from eight primary schools classes $(N=139)$. It was one purpose of the study to replicate and systematize former results concerning the difficulty of word problems. Further should be examined, if the preceding processing of change or equalizing word problems facilitates the solution of comparison word problems. Results indicate that students do not use various linguistic realizations of the same mathematical structure for solving word problems without explicit instruction. This leads to the question, in which way explicit training could support the students with the reinterpretation of such word problems.
\end{abstract}

Keywords Flexibility $\cdot$ semantic structure $\cdot$ additive word problems $\cdot$ comparison of sets

\section{Stand der Forschung}

\subsection{Sprachliche Kompetenzen und mathematische Lernprozesse}

Spätestens seit den Ergebnissen von PISA, TIMSS und IGLU ist auch der breiteren Öffentlichkeit bekannt, dass ein Zusammenhang zwischen Mathematik- und 
Sprachkompetenzen besteht. Den Ergebnissen zufolge sind Lernende mit Migrationshintergrund diesbezüglich besonders benachteiligt (z. B. Baumert und Schümer 2001; Wendt et al. 2016). In neueren Untersuchungen lassen sich beispielsweise Leistungsnachteile von Lernenden mit nicht-deutscher Familiensprache, die im Fach Mathematik bereits im ersten Schuljahr bestehen (Heinze et al. 2007), zu einem großen Teil durch Sprachkompetenzen erklären (Ufer et al. 2013). Sprachliche Kompetenzen stellen sich beim mathematischen Kompetenzerwerb allerdings nicht nur für Lernende mit nicht-deutscher, sondern auch für Lernende mit deutscher Familiensprache als relevant heraus (Paetsch et al. 2016). Damit wird der Fokus auf alle Lernenden mit niedrigen Sprachkompetenzen erweitert. In bisherigen Studien werden sprachliche Kompetenzen relativ breit operationalisiert. Als übergeordnetes Konstrukt werden diese benötigt, um in unterschiedlichen Situationen sprachliche Anforderungen zu bewältigen (Klieme 2004).

Eine Ursache für sprachbedingte Unterschiede in mathematischen Leistungen könnte in den spezifischen sprachlichen Anforderungen meist schriftlicher Testsituationen liegen (Haag et al. 2013). Allerdings konnte gezeigt werden, dass eine gezielte sprachliche Vereinfachung mathematischer Testaufgaben, z. B. durch Vereinfachung des Vokabulars oder der Grammatik, nicht speziell für Lernende mit niedrigen Sprachkompetenzen, sondern für alle Lernenden gleichermaßen wirksam ist (Abedi et al. 2006). Es ist also eher unwahrscheinlich, dass die beobachteten sprachbedingten Leistungsunterschiede im Fach Mathematik allein durch die sprachlichen Anforderungen in Testsituationen zustande kommen. Um die Rolle von Sprache für den mathematischen Kompetenzerwerb zu erklären, müssen deshalb weitere Wirkmechanismen herangezogen werden.

Eine weitere Vermutung bezieht sich auf die Verwendung von Sprache im Unterrichtsdiskurs (Civil 2008). Auch wenn Mathematik aufgrund einer intensiven Nutzung von Symbolen häufig als spracharm wahrgenommen wird, muss im Unterricht dennoch die Bedeutung der spezifischen Symbole und Darstellungen sprachlich ausgehandelt werden. Wie Schütte (2009) in seiner Dissertation an Interaktionsanalysen illustrieren konnte, erfolgt dieser Aushandlungsprozess meist implizit, ihm zu folgen ist daher häufig anspruchsvoll. Qualitative Studien aus dem US-amerikanischen Kontext weisen darauf hin, dass es Lernenden mit niedrigeren sprachlichen Kompetenzen teilweise schwerfällt, die Äußerungen ihrer Lehrkräfte sprachlich zu verstehen (Civil 2008). Auch die Möglichkeiten zum Austausch mit anderen Lernenden können eingeschränkt sein (Moschkovich 2007). Eine Längsschnittstudie von Bochnik (2017) belegt, dass der Kompetenzzuwachs besonders bei solchen Lernenden ausgeprägter war, die berichteten, dem Unterrichtsgespräch sprachlich gut folgen zu können. Diese Befunde liefern erste Hinweise, dass die sprachliche Interaktion im Unterricht ein wesentlicher Vermittler zwischen Sprachkompetenz und mathematischem Lernerfolg ist.

Darüber hinaus zielt ein weiterer Erklärungsansatz auf die kognitive Nutzung von Sprache ab. Sprache kann als Werkzeug zur Strukturierung und Konstruktion mathematischen Wissens dienen (Sfard 2008; Steenpaß und Steinbring 2014). Geringe sprachliche Kompetenzen können eine Hürde beim kognitiven Erfassen fachlicher Zusammenhänge darstellen, da ohne sprachliches Repertoire kognitive Repräsentationen nur unvollständig aufgebaut werden können und weitere kognitive 
Operationen wie Transferleistungen oder Problemlösen nur eingeschränkt möglich sind (Kempert et al. 2018). Diese Funktion von Sprache spielt beispielsweise bei der Bearbeitung von Textaufgaben eine zentrale Rolle: Um die in der Textaufgabe dargestellte Situation zu verstehen und zu modellieren, müssen mathematische Strukturen aus der verbalen Beschreibung erschlossen werden. Der vorliegende Beitrag fokussiert im Sinne dieses letztgenannten Erklärungsansatzes, wie Lernende mit der sprachlichen Darstellung mathematischer Zusammenhänge in Textaufgaben umgehen.

Im Beitrag wird anhand von Textaufgaben die Rolle von Flexibilität im Umgang mit Situationsstrukturen für die Nutzung sprachlicher Beschreibungen mathematikhaltiger Situationen fokussiert. Damit beschäftigt sich der Beitrag weniger mit den individuellen sprachlichen Fähigkeiten der Lernenden und deren Bedeutung für das mathematische Lernen und Arbeiten, sondern primär mit Strategien und Ressourcen (nicht unbedingt sprachlicher Natur), die Lernende nutzen können, um mit der sprachlichen Komplexität (z.B. von Textaufgaben) umzugehen. Zunächst werden Modelle zum Lösen von additiven ${ }^{1}$ Textaufgaben vorgestellt. Die bestehenden Modelle zu individuellen Bearbeitungsprozessen beim Lösen solcher Textaufgaben werden in ein alternatives Modell zusammengeführt, das die in der Textaufgabe dargestellte Situation nach Eigenschaften auf verschiedenen Ebenen differenziert. Aus typischen Hürden während des Bearbeitungsprozesses und der Literatur zu diesen Schwierigkeiten werden Ansatzpunkte für ein Förderkonzept abgeleitet. Dazu wird die Förderung einer Flexibilität im Umgang mit mathematischen Situationsstrukturen, welche die Umdeutung von schwierigeren Textaufgaben in leichtere ermöglichen soll, als Ansatzpunkt für Interventionen vorgeschlagen. Anschließend werden die Methodik und Ergebnisse einer Studie vorgestellt, die wesentliche Fragen zur Konzeption eines auf diesem Ansatz basierenden Förderkonzepts bearbeitet.

\subsection{Lösen von Textaufgaben als Rekonstruktion und Anreicherung von Situationsstrukturen}

Textaufgaben sind aus mathematikdidaktischer Perspektive von Sachaufgaben abzugrenzen: Während Sachaufgaben auf die Bewältigung von Mathematik in konkreten, komplexeren Alltagssituationen abzielen, steht bei der Bearbeitung von Textaufgaben die Vermittlung mathematischer Konzepte im Vordergrund (Stern 1998, S. 84 f.). Sie dienen also im Unterricht primär dem Zweck, die verschiedenen mit einem mathematischen Konzept verbundenen Situationsstrukturen und Möglichkeiten für deren sprachliche Realisierung zu thematisieren. In vorliegenden Arbeiten werden oft ausschließlich sogenannte Simplexaufgaben (Franke und Ruwisch 2010, S. 12 f.; Breidenbach 1969, S. $180 \mathrm{f}$.) betrachtet, die mit einer einzelnen Rechenoperation lösbar sind und keine fehlenden oder überflüssigen Angaben enthalten. Vorteil einer

\footnotetext{
1 Der Literatur (z. B. Verschaffel et al. 2020) folgend verwenden wir den Begriff ,additiv“ für Situationen und Situationsstrukturen als Oberbegriff für Situationen, die mit einer Addition oder Subtraktion modelliert werden können. Für mathematische Modelle differenzieren wir im Text jedoch zwischen ,,additiven Strukturen bzw. Modellen“ (die eine Addition als Operation enthalten, z. B. auch als Teil des Ergänzens) und ,subtraktiven Strukturen bzw. Modellen“ (die eine Subtraktion als Operation enthalten).
} 


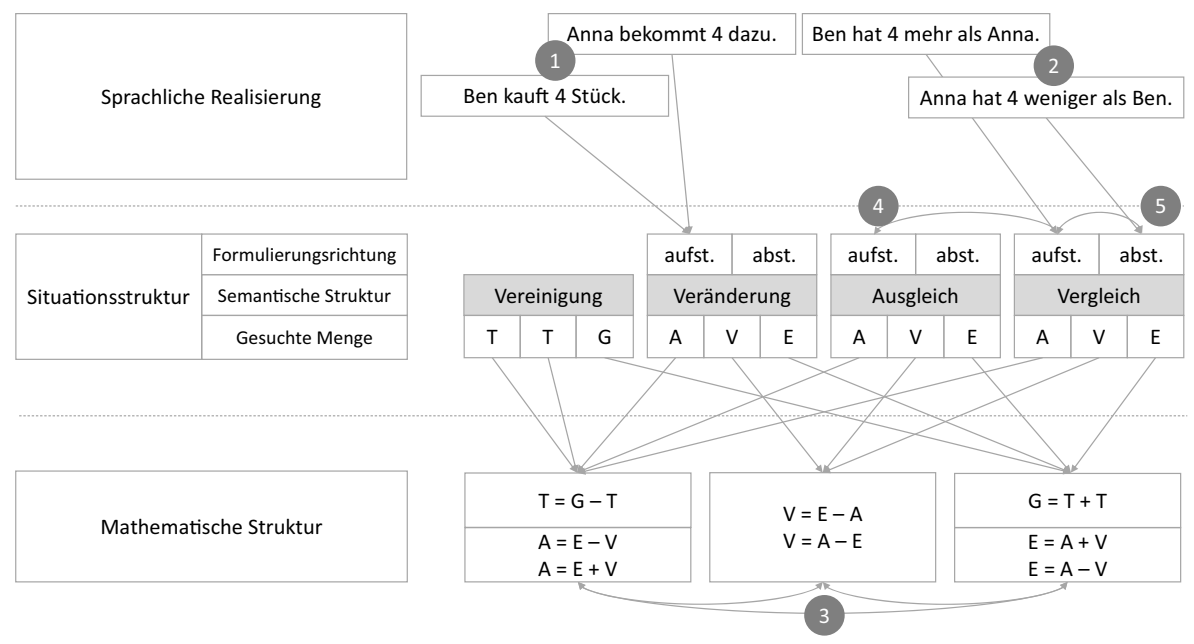

Abb. 1 Modell der Strukturebenen von Textaufgaben ( $T$ Teilmenge, $G$ Gesamtmenge, $A$ Ausgangsmenge, $V$ Veränderungsmenge, $E$ Ergebnismenge)

solchen Fokussierung ist die Möglichkeit, Schwierigkeitsmerkmale der Aufgaben gezielt zu kontrollieren. Die so gewonnenen Ergebnisse lassen dadurch jedoch keine direkten Schlüsse über alle Teilprozesse beim Lösen von komplexeren Sachaufgaben zu.

Bezüglich des Lösens von Textaufgaben im schulischen Kontext gibt es national sowie international eine lange Forschungstradition (Kintsch und Greeno 1985; Stern 1998; Vicente et al. 2008). Gängige Modelle der Bearbeitungsprozesse beim Lösen von Textaufgaben (z.B. Kintsch und Greeno 1985) beschreiben dabei neben dem konkreten Aufgabentext zwei verschiedene mentale Modelltypen, die individuell aufgebaut werden müssen: Das Situationsmodell und das mathematische Problemmodell. Diesen individuell von den Lernenden konstruierten Modellen gegenüber stehen die Strukturen, die beim Erstellen der Textaufgabe von der Autorin bzw. vom Autor intendiert wurden (siehe Abb. 1, Erläuterung im Folgenden). Das Modell in Abb. 1 erlaubt dementsprechend zwei Sichtweisen: Einerseits auf die von der Autorin bzw. vom Autor intendierten Strukturen (exemplarisch siehe Anhang, Abb. 3), andererseits auf mögliche von Lernenden individuell als Teil des Situationsmodells rekonstruierte Strukturen (exemplarisch siehe Anhang, Abb. 4). Das Modell unterscheidet die Situationsstrukturen und die mathematischen Strukturen, die von der Autorin bzw. vom Autor bei der Beschreibung der Situation intendiert wurden. Diese Strukturen werden von Lernenden beim Lösen einer Textaufgabe so gut wie möglich dekodiert, indem ein individuelles Situationsmodell und ein individuelles mathematisches Problemmodell gebildet werden. Modellhaft können zwei individuelle Teilprozesse unterschieden werden, durch die diese Strukturen beim Lösen der Textaufgabe von den Lernenden mehr oder weniger vollständig rekonstruiert werden: 
1. Die in der Textaufgabe dargestellte Situationsstruktur kann zunächst auf unterschiedliche Arten sprachlich als Text realisiert (Ebene sprachliche Realisierung in Abb. 1) sein. In einer ersten Phase wird dieser Aufgabentext sprachlich entschlüsselt und unter Einbezug des individuellen Vorwissens in ein initiales Situationsmodell integriert. Dieses enthält im besten Fall die wesentlichen dargestellten Komponenten der Situation und die wesentlichen dargestellten Zusammenhänge, also bestimmte Aspekte der Situationsstruktur (siehe Abb. 1; Ebene Situationsstruktur). Dabei können für die Lösung als unwichtig erachtete Informationen aus dem Text ausgeblendet und nicht direkt im Text enthaltene, zur Bearbeitung notwendige Informationen gegebenenfalls ergänzt werden (Stern 1998, S. 104). Modellen des Leseverstehens (z. B. Kintsch 1998, S. 92 ff.) zufolge werden die Inhalte des Aufgabentextes dabei nicht einfach mental rekonstruiert, sondern auch durch weitere Inferenzen angereichert, die diese Inhalte auf der Basis individuellen Vorwissens um weitere Eigenschaften der Situation ergänzen (z. B. weitere Aspekte der Situationsstruktur, Abb. 1, (4) und (5); s. a. Abschn. 1.4 unten). Die von Lernenden dekodierte Situationsstruktur wird also zu Beginn des Bearbeitungsprozesses bestenfalls mit der im Aufgabentext beschriebenen Situationsstruktur übereinstimmen. Sie kann jedoch im weiteren Verlauf durch Inferenzen weiter ausdifferenziert und angereichert werden. In diesem Sinne könnten beispielsweise Vergleichssituationen in Ausgleichssituationen umgedeutet werden, indem eine Handlung, die den beschriebenen Unterschied ausgleichen würde, in das Situationsmodell aufgenommen wird.

Wie eine Situation in einer schriftlichen Textaufgabe konkret in Textform dargestellt wird, kann dabei auf zwei substantiell unterschiedlichen Ebenen variieren. Zunächst kann der Text unterschiedliche strukturelle Eigenschaften einer Situation hervorheben, um diese sprachlich zu beschreiben (Situationsstruktur in Abb. 1, Anhang Abb. 3). Beispielsweise kann die Tatsache, dass Ben vier Kekse mehr als Anna hat, auch dadurch beschrieben werden, dass Anna genauso viele Kekse wie Ben hätte, wenn sie noch vier Kekse (von jemand anderem) bekommen würde oder, dass Anna vier Kekse weniger hat als Ben. Für viele mathematische Konzepte wurden verschiedene Typen solcher semantischen Strukturen, die durch dieselben mathematischen Strukturen und Konzepte beschrieben werden können, aus sachanalytischer Sicht klassifiziert (Schipper 2009; unter dem Begriff „Grundvorstellung": vom Hofe 1995; unter dem Begriff ,semantische Struktur“: Radatz 1983). So werden beispielsweise für arithmetische Operationen dynamische Veränderungen und statische Vergleiche unterschieden (Radatz 1983; Schmidt 2009), oder für Funktionen Zuordnungs- und Kovariationssituationen (Vollrath 1989).

Neben der Frage, anhand welcher Eigenschaften eine Situation dargestellt wird, kann diese durch unterschiedliche sprachliche Mittel realisiert werden (sprachliche Realisierung in Abb. 1). Einerseits erfordert die Beschreibung bestimmter semantischer Strukturen bestimmte sprachliche Mittel, z. B. erfordert die Darstellung des Vergleichs zweier Mengen Relationsbegriffe wie mehr, weniger, größer oder kleiner. Andererseits können unterschiedliche Sprachregister zur Darstellung herangezogen werden, beispielsweise alltagssprachliche, bildungssprachliche oder fachsprachliche Register (Prediger und Wessel 2013). 
Auf individueller Ebene ist es plausibel, dass unterschiedliche Wissensfacetten notwendig sind, um ein Situationsmodell aufzubauen. Beispielsweise erfordert eine Dekodierung der sprachlichen Realisierung allgemeinsprachliche und fachsprachliche Kompetenzen (Ufer und Bochnik 2020). In der Tat fasst Paetsch (2016) einige Studien zusammen, die den Zusammenhang von sprachlichen Fähigkeiten und der Leistung beim Lösen von Textaufgaben belegen. Der Aufbau eines Situationsmodells erfordert zudem jedoch individuelles Wissen zu typischen additiven Situationen, das für diesen Teilprozess zunächst weder zwingend mit mathematischem Wissen verknüpft sein muss, noch eine starke Vernetzung verschiedener Situationstypen beinhalten muss.

2. Mit der von der Autorin bzw. vom Autor der Textaufgabe intendierten Situationsstruktur sind meist eine oder mehrere intendierte mathematische Strukturen verbunden. Auf individueller Ebene muss das konstruierte Situationsmodell im weiteren Verlauf des Lösungsprozesses in ein mathematisches Problemmodell übertragen werden; das heißt, es wird mittels mathematischer Konzepte beschrieben (Kintsch und Greeno 1985). Im besten Falle gibt das so generierte mathematische Problemmodell die in der Textaufgabe intendierten mathematischen Strukturen (s. Abb. 1) korrekt wieder. Diese Auswahl einer bestimmten mathematischen Struktur als individuelles Problemmodell basiert einerseits auf dem individuellen Situationsmodell, welches mehr oder weniger umfassend die relevanten Aspekte der Situationsstruktur enthalten kann. Andererseits wird dafür individuelles Wissen benötigt, durch welche mathematische(n) Struktur(en) eine bestimmte Situationsstruktur beschrieben werden kann. Dieses Wissen um mögliche Bedeutungen bestimmter mathematischer Strukturen und Konzepte wird in der Mathematikdidaktik ebenfalls mit dem Begriff „Grundvorstellungen“ beschrieben (Blum und Leiß 2005; vom Hofe 1995). In psychologischen Modellen werden Schematheorien verwendet, um die mentale Repräsentation von Situationsstrukturen (als Situationsmodelle) und Lösungsstrategien (als mathematische Problemmodelle) zu beschreiben (Kintsch und Greeno 1985). Gemeinsam ist beiden Perspektiven, dass sie davon ausgehen, dass unterschiedliche „Schemata“ (bzw. „Grundvorstellungen") individuell verfügbar sein müssen und auch aktiviert werden müssen, um unterschiedlich strukturierte Situationsmodelle zu mathematisieren. Es werden beispielsweise Grundvorstellungen zu Vergleichs- und Veränderungssituationen unterschieden (Schipper 2009), oder sogenannte Compare- und Change-Schemata (Riley et al. 1983; Schipper 2009, S. 99), die jeweils die entsprechenden Situationsstrukturen repräsentieren (semantische Struktur in Abb. 1).

Zusammenfassend heben die beschriebenen Modelle hervor, dass das aus der sprachlichen Realisierung im Aufgabentext rekonstruierte, mehr oder weniger umfassende Situationsmodell wesentlich für die Verarbeitung von Textaufgaben ist. Je nachdem, welche Aspekte der Situationsstruktur im individuellen Situationsmodell verfügbar sind, können unterschiedliche Grundvorstellungen bzw. Schemata aktiviert werden, um ein mathematisches Problemmodell zu generieren. Trotz der modellhaften Trennung der beiden Phasen wird in der Literatur jedoch herausgestellt, dass sprachliches, situationsbezogenes und mathematisches Wissen während des 
gesamten Bearbeitungsprozesses nicht getrennt voneinander aktiviert wird, sondern miteinander interagiert (Stern 1998, S. 106).

Dieser Beitrag widmet sich besonders der Frage, was passiert, wenn Lernende die von ihr direkt aus dem Text identifizierte Situationsstruktur nicht mit einer mathematischen Struktur in Verbindung bringen können. Im Folgenden wird argumentiert, dass in diesem Fall eine Umdeutung der Situationsstruktur (Abb. 1, exemplarisch Pfeile (4) und (5) eine alternative Strategie zur Identifikation einer passenden mathematischen Struktur sein kann. Im Fokus des Beitrags steht, in welchem Umfang Lernende eine solche Strategie spontan nutzen, um mit empirisch schwierigeren sprachlichen Beschreibungen mathematischer Strukturen umzugehen.

\subsection{Schwierigkeitsunterschiede bei additiven Textaufgaben}

Bislang wurde unter anderem untersucht, welche Faktoren das Lösen einer Textaufgabe erschweren. Befunde zu sprachlichen Merkmalen von Mathematikaufgaben weisen darauf hin, dass die konkrete sprachliche Realisierung der in einer Textaufgabe beschriebenen Situation, wie z.B. das jeweilige Sprachregister (Prediger und Wessel 2013), die Schwierigkeit einer Aufgabe beeinflussen können (siehe z.B. Bailey und Butler 2003). Diese Ebene der sprachlichen Realisierung steht nicht im Fokus dieses Beitrags und wurde in der vorliegenden Studie so weit möglich kontrolliert. Neben der konkreten sprachlichen Realisierung hat es sich als relevant erwiesen, welche strukturellen Eigenschaften der Situation in der Textaufgabe beschrieben werden. Unterschiedliche reale Situationen (siehe Bsp. (1) in Abb. 1) können dabei die gleiche Situationsstruktur realisieren. Verschiedene sprachliche Formulierungen (siehe Bsp. (2) in Abb. 1) können jedoch auch unterschiedliche Situationsstrukturen zur gleichen realen Situation hervorheben. Im Folgenden werden verschiedene Komponenten der Situationsstruktur einer Textaufgabe (siehe Abb. 1) und in diesem Zusammenhang Befunde zu Schwierigkeitsunterschieden vorgestellt.

\subsubsection{Semantische Struktur}

Die semantische Struktur beschreibt, welche Beziehungen zwischen den in der Textaufgabe behandelten Größen beschrieben bzw. in ein individuelles Situationsmodell übernommen werden. Beim Aufbau eines mathematischen Problemmodells wird das Verständnis dieser semantischen Struktur als ein wesentlicher Faktor für Schwierigkeitsunterschiede zwischen verschiedenen Textaufgaben diskutiert. Dabei werden als semantische Strukturen in additiven Textaufgaben häufig Veränderungs-, Vereinigungs-, Vergleichs- oder Ausgleichsaufgaben unterschieden (Riley et al. 1983; Schipper 2009, S. 99). Eine Übersicht dazu findet sich in Tab. 1.

Während Veränderungs- und Ausgleichsaufgaben als dynamisch bezeichnet werden, da sie eine Handlung beinhalten, gelten Vereinigungs- und Vergleichsaufgaben als statisch (Riley et al. 1983; Carpenter et al. 1981). Unterscheidet man wiederum nach Beziehungen zwischen den vorkommenden Mengen, können die vier semantischen Strukturen entweder einer Teil-Teil-Ganzes-Beziehung (Veränderung, Vereinigung) oder disjunkten Mengen (Ausgleich, Vergleich) zugeordnet werden (Radatz et al. 1996). Bezüglich der Schwierigkeit dieser unterschiedlichen semantischen 
Tab. 1 Beispielaufgaben zu semantischen Strukturen von additiven Textaufgaben mit Bezeichnungen der beteiligten Mengen

\begin{tabular}{lll}
\hline & Dynamische Situation & Statische Situation \\
\hline $\begin{array}{l}\text { Teil- } \\
\text { Teil- }\end{array}$ & Veränderung & Vereinigung \\
Ganzes- & Anna hat 3 Murmeln (A). & Anna hat 3 Murmeln (T), Ben hat \\
Struktur & Dann bekommt sie 5 Murmeln (V) dazu. & 5 Murmeln (T). \\
& Wie viele Murmeln hat Anna jetzt (E)? & Wie viele Murmeln haben sie \\
Disjunkte & Ausgleich & zusammen (G)? \\
Mengen & Anna hat 3 Murmeln (A). & Vergleich \\
& Wenn sie 5 Murmeln (V) dazubekommt, dann hat & Anna hat 3 Murmeln (A). \\
& sie genauso viele wie Ben. & Ben hat 5 Murmeln (V) mehr als \\
& Wie viele Murmeln hat Ben (E)? & Sie.
\end{tabular}

$T$ Teilmenge, $G$ Gesamtmenge, $A$ Ausgangsmenge, $V$ Veränderungsmenge, $E$ Ergebnismenge

Strukturen zeigen zahlreiche Studien ein relativ einheitliches Bild: Während Veränderungs- und Vereinigungsaufgaben zu den einfacheren semantischen Strukturen gehören, stellen besonders Vergleichsaufgaben für viele Lernende eine Herausforderung dar (Riley und Greeno 1988; z. B. Cummins et al. 1988; Stern 1992). Das wird unter anderem damit begründet, dass die im Vergleich enthaltene Veränderungsmenge keine in der Situation wirklich existierende Menge, sondern eine Beziehung zwischen zwei Mengen beschreibt und somit für Lernende schwerer vorstellbar ist (Stern 1994). Auch aktuelle Modelle des Zahlbegriffserwerbs ordnen diese semantischen Strukturen unter dem Begriff ,Relationalität“ in spätere Phasen der Entwicklung zum Schulbeginn ein (im Überblick z. B. Fritz et al. 2018). Ausgleichsaufgaben wurden bisher seltener untersucht, da sie in vielen Studien nicht aufgenommen bzw. nicht als eigene semantische Struktur unterschieden wurden. Die bei Stern (1994) als Angleichungsaufgaben bezeichneten Ausgleichsaufgaben wurden von Lernenden der ersten Jahrgangsstufe zu durchschnittlich $96 \%$ gelöst.

\subsubsection{Gesuchte Menge}

Aufgaben zu jeder semantischen Struktur können außerdem in Bezug auf die gesuchte Menge variieren (siehe auch Abb. 1). In einer Simplexaufgabe geht es um jeweils drei Mengen, von welchen zwei bekannt sind und die dritte Menge mit deren Hilfe berechnet werden soll (Stern 1998). Solche Aufgaben stellen sich als einfacher heraus, wenn je nach semantischer Struktur die Gesamt- (Vereinigung) oder Ergebnismenge (Veränderung, Ausgleich, Vergleich) gesucht ist ${ }^{2}$. Eher geringe Lösungsraten erzielen Lernende bei Vergleichsaufgaben mit fehlender Ausgangsmenge (bei Stern Referenzmenge). Als Ursache dafür werden häufig Signalwortstrategien genannt, bei welchen von der Formulierung der Aufgabe auf die auszuführende Rechenoperation geschlossen wird (Fuson et al. 1996). Beispielweise könnte das

\footnotetext{
2 Die Bezeichnungen für die Mengen variieren in der Literatur abhängig von der semantischen Struktur. Zur einfacheren Orientierung haben wir für den vorliegenden Beitrag einheitliche Begriffe gewählt (s.a. Tab. 2 im Anhang).
} 
Wort weniger bei der Aufgabe „Anna hat 3 Nüsse. Sie hat 2 Nüsse weniger als Ben. Wie viele Nüsse hat Ben?“ zur Ausführung einer Subtraktion verleiten. Besser gelöst wurden Vergleichsaufgaben mit fehlender Ergebnismenge, wie z.B.: „Anna hat 3 Nüsse. Ben hat 2 Nüsse mehr als Anna. Wie viele Nüsse hat Ben?“.

\subsubsection{Formulierungsrichtung}

Vor dem Hintergrund von Signalwortstrategien wird auch die Formulierungsrichtung (absteigend bzw. aufsteigend) als weiterer Einflussfaktor diskutiert (z. B. Fuson et al. 1996) (vgl. Abb. 1). Bei Veränderungs- und Ausgleichsaufgaben werden unterschiedliche Richtungen der konkreten Handlungen bzw. unterschiedliche Sichtweisen auf eine Situation für die sprachliche Beschreibung herausgegriffen. Damit werden einzelne strukturelle Eigenschaften der Situation hervorgehoben, andere weggelassen. Durch die sprachliche Realisierung einer Situation (z. B. in Form einer Textaufgabe) wird nur ein bestimmter Ausschnitt der möglichen Situationsstrukturen gezeigt. Daher ordnen wir aufsteigende und absteigende Formulierungen zu Veränderungsund Ausgleichsaufgaben nicht der Ebene der sprachlichen Realisierung, sondern der Situationsstruktur zu. Diese auf- bzw. absteigende Richtung zeigt sich wiederum in der sprachlichen Realisierung von Veränderungs- und Ausgleichsaufgaben durch Handlungsverben zum Hinzufügen (z. B. dazubekommen, kaufen ...) und Wegnehmen (z. B. abgeben, verschenken ...). Diese als invers zueinander zu erkennen ist ein Inferenzprozess, der über den Inhalt des Aufgabentextes hinausgeht. Vergleichsaufgaben können mithilfe von Relationsbegriffen ebenso aufsteigend (mehr als, höher als ...) oder absteigend (weniger als, kürzer als ...) formuliert werden und legen damit unterschiedliche Sichtweisen auf eine Situation fest. Die dazugehörige symmetrische Relationsaussage bleibt zunächst verborgen und kann erst beim Erschließen der Situationsstruktur ergänzt werden. Auch wenn die spezifische Formulierung der Situation prinzipiell austauschbar ist, scheint gerade der für das Erkennen dieser Äquivalenz notwendige Inferenzprozess eine wesentliche Herausforderung beim Umgang mit solchen Situationsstrukturen zu sein. Vereinigungsaufgaben werden in der Literatur nicht in ihrer Formulierungsrichtung variiert.

Unabhängig von der möglichen Erklärung durch eine Signalwortstrategie weisen die genannten Ergebnisse darauf hin, dass Textaufgaben leichter sind, wenn die Formulierungsrichtung (aufsteigend bzw. absteigend) konsistent mit der Operation (Addition bzw. Subtraktion) ist, die direkt zur Lösung der Aufgaben angewendet werden kann. Einschränkend kann angemerkt werden, dass beispielsweise auch die additive Strategie „Ergänzen“ als mathematisch äquivalente Strategie zur Subtraktion zur Lösung herangezogen werden könnte (siehe Bsp. (3) in Abb. 1). Torbeyns et al. (2009) argumentieren jedoch, dass diese Strategie eher selten spontan angewendet wird.

Stern (1993) hat in einer Studie die Verwendung der Signalwortstrategie als Einflussfaktor auf die Lösung von additiven Textaufgaben untersucht, indem die Lernenden verschiedene Vergleichsaufgaben nacherzählen sollten. Dabei stellte sich heraus, dass Vergleichsaufgaben mit fehlender Ergebnismenge besser nacherzählt werden konnten als solche mit fehlender Ausgangsmenge. Daraus schließt Stern (1993), dass Signalwortstrategien nicht die alleinige Ursache für unterschiedliche 
Lösungsraten sein können. Sie weist in ihren Untersuchungen auf einen weiteren möglichen Einflussfaktor hin und nennt in diesem Zusammenhang das Verständnis der sprachlichen Symmetrie eines quantitativen Vergleichs. Ergebnisse der Studie von Stern (1993) weisen darauf hin, dass $70 \%$ der teilnehmenden Lernenden der ersten Jahrgangsstufe die Äquivalenz der Aussagen „Anna hat 2 Nüsse weniger als Ben“ und „Ben hat 2 Nüsse mehr als Anna“ nicht nachvollziehen konnten und damit wenig Flexibilität im Umgang mit der Formulierungsrichtung einer Textaufgabe zeigten. Dieses Verständnis wäre allerdings zur Lösung schwieriger Vergleichsaufgaben mit fehlender Ausgangsmenge (,Anna hat 5 Nüsse. Anna hat 2 Nüsse weniger als Ben.") hilfreich, da mit diesem Wissen eine solche Aufgabe in die (empirisch leichtere) Vergleichsaufgabe mit fehlender Ergebnismenge (,Anna hat 5 Nüsse. Ben hat 2 Nüsse mehr als Anna.") umgedeutet werden könnte. In der Literatur nicht benannt, aber ebenfalls denkbar ist, dass auch die Beziehung zwischen auf- und absteigenden Veränderungen in ähnlicher Weise genutzt werden könnte. Vor diesen Ergebnissen erscheint es plausibel, dass Signalwortstrategien weniger das ursächliche Problem beim Lösen von Textaufgaben sind, sondern eher einen Hinweis auf fehlendes konzeptuelles Wissen zu additiven Situationsstrukturen, das für alternative und tragfähigere Strategien genutzt werden könnte, geben. Offen bleibt zunächst, ob dabei - wie Stern annimmt - vorwiegend Wissen zur Symmetrie quantitativer Vergleiche, Wissen um den Relationszahlaspekt natürlicher Zahlen (Fritz et al. 2018) oder meist in der Praxisliteratur genannte, allgemeiner ausgeprägte Grundvorstellungen zu additiven Situationsstrukturen (z. B. Fromme et al. 2011) relevant sind.

Zusammenfassend liegen zur Schwierigkeit von Textaufgaben zahlreiche, relativ einheitliche Resultate vor. Als relevant für die Schwierigkeit der Aufgaben auf der Ebene der abgebildeten Situationsstruktur hat sich herausgestellt, (i) welche semantische Struktur der Textaufgabe zugrunde liegt, (ii) in welcher Richtung diese Struktur beschrieben wird (aufsteigend vs. absteigend) und (iii) welche Menge in der beschriebenen Situation gesucht ist (siehe Ebene der Situationsstruktur in Abb. 1). Insgesamt sprechen insbesondere (ii) und (iii) dafür, dass vor allem relevant ist, welche Aspekte der Situationsstruktur im Aufgabentext präsentiert werden und weniger, anhand welcher Begrifflichkeiten dies geschieht. Entsprechend geht der Beitrag von der Annahme aus, dass weniger die Dekodierung des Aufgabentextes (Schritt von der sprachlichen Realisierung hin zur Situationsstruktur) für diese Schwierigkeitsunterschiede ursächlich ist, sondern primär die Zuordnung einer mathematischen Struktur zu einer identifizierten Situationsstruktur.

Methodisch ist zu den genannten Studien anzumerken, dass in der Regel gemeinsam mit den genannten Aufgabenmerkmalen auch weitere Merkmale wie die in den Aufgaben enthaltenen Namen, Objekte und das konkrete Zahlenmaterial variiert wurden. Auch wenn hier nur geringe Effekte anzunehmen sind, steht eine Abschätzung der dadurch hervorgerufenen Unterschiede jedoch noch aus. Zentraler erscheint jedoch, dass sich der Mathematikunterricht - auch im Rahmen der Diskussion zu prozessbezogenen Kompetenzen (Klieme et al. 2003), zum Modellieren (Greefrath et al. 2013) und zur Rolle von Textaufgaben (Verschaffel et al. 2000) - seit diesen Studien deutlich weiterentwickelt haben könnte. Daher besteht die Notwendigkeit einer Überprüfung und darüber hinaus einer Systematisierung der vorliegenden Befunde. 


\subsection{Flexibilität im Umgang mit mathematischen Situationsstrukturen als Interventionsansatz}

Die teilweise großen Unterschiede in den Lösungsraten zu Textaufgaben mit unterschiedlicher Situationsstruktur führten bereits sehr früh zu Ideen, ob sich Leistungen bei einfacheren Aufgabentypen nicht nutzbar machen lassen, um schwierigere Aufgabentypen zu erschließen. So schlägt Greeno (1980) in Bezug auf semantische Strukturen vor, dass schwierigere Veränderungsaufgaben in Vereinigungsaufgaben umgedeutet werden könnten. Anstatt beispielsweise die Aufgabe ,Jill hatte drei (A) Äpfel. Betty gab ihr einige dazu. Jetzt hat Jill acht (E) Äpfel. Wie viele Äpfel hat Betty ihr gegeben?“ über eine Veränderungsstruktur mit gegebener Anfangs- (A) und Ergebnismenge (E) zu lösen, beschreibt Greeno die Möglichkeit, die Struktur der Aufgabe in eine Vereinigungsstruktur mit drei als Teilmenge (T) und acht als Gesamtmenge (G) umzudeuten. Ein Beispiel könnte lauten: ,Jill hat drei (T) grüne Äpfel und dazu noch einige rote (die Betty ihr gegeben hat). Insgesamt hat sie acht (G) Äpfel. Wie viele rote Äpfel hat sie (die sie von Betty bekommen hat)?“.

In Modellen zum Lösen von Textaufgaben ließen sich diese Strategien als Inferenzprozess beim Lesen deuten, der die im Text beschriebene Situationsstruktur durch eine alternative Sichtweise auf die Situation ergänzt.

Angesichts der in der Literatur dokumentierten Schwierigkeit von Vergleichsaufgaben wäre zu überlegen, ob diese in ähnlicher Weise in Aufgaben mit einer anderen semantischen Struktur umgedeutet werden könnten. Die Arbeit von Greeno (1980) beschäftigte sich vorwiegend mit der Simulation menschlichen Verhaltens durch Computersysteme. Auf menschliche Lernende übertragen stellt sich die Frage, warum diese die Strategie, schwierige semantische Strukturen in einfache umzudeuten, bei Vergleichsaufgaben nicht spontan anwenden und ob bzw. unter welchen Bedingungen eine solche Strategie hilfreich sein könnte. Besonders naheliegend scheint hier die Umdeutung von statischen Vergleichsaufgaben (Jill hat fünf Äpfel mehr als Betty) in dynamische Ausgleichsaufgaben (Wenn Betty noch fünf Äpfel dazubekommt, dann hat sie genauso viele wie Jill; vgl. Abb. 1, (4)), da diese Situationen beide disjunkte Mengen enthalten, Textaufgaben zu Ausgleichsaufgaben allerdings in Stufenmodellen zu additiven Textaufgaben auf einem niedrigeren Niveau als Vergleichsaufgaben eingestuft werden (Radatz 1983; Nesher et al. 1982).

Stern (1993) regt ebenfalls an, Umdeutungen von Textaufgaben als Lösungsstrategie zu nutzen. Sie diskutiert allerdings keine Beziehungen zwischen Situationen zu unterschiedlichen semantischen Strukturen, sondern geht auf die Rolle der Formulierungsrichtung ein. Sie spricht von der sprachlichen Symmetrie quantitativer Vergleiche und weist hier explizit auf die notwendige Flexibilität im Umgang mit der Formulierungsrichtung quantitativer Vergleiche hin (vgl. Abschn. 1.3). Ausgehend von ihren Resultaten zur Rolle der Formulierungsrichtung wäre ein weiterer Interventionsansatz, die Symmetrie quantitativer Vergleiche anhand von Beispielen (z.B. Anna hat 2 Nüsse weniger als Ben, Ben hat 2 Nüsse mehr als Anna) mit den Lernenden explizit zu thematisieren. Dies würde also auf den Aufbau einer anderen Umdeutungsstrategie bei den Lernenden abzielen: Flexibilität im Umgang mit der Formulierungsrichtung soll Lernende einerseits für die Rolle der Formulierungsrichtung und der gesuchten Menge sensibilisieren, es ihnen andererseits erleichtern, 
Textaufgaben so umzudeuten, dass die Operation mit der sprachlichen Beschreibung ihres Situationsmodells übereinstimmt. Auch dieser Ansatz schlägt einen zusätzlichen Inferenzprozess beim Lesen der Textaufgabe vor, der das Situationsmodell anreichert und der in diesem Fall zu einer alternativen Sichtweise auf die Relationsstruktur im Situationsmodell führt (vgl. Abb. 1, (5)).

Beide Ansätze zielen also darauf ab, dass Lernende ein auf der Basis des Aufgabentextes erstelltes Situationsmodell durch zusätzliche Inferenzen im Leseprozess erweitern, und so die dargestellte Situation in eine Situation mit einer anderen (einfacher mathematisierbaren) Struktur umdeuten. Ein Beispiel dafür ist im Anhang (siehe Anhang, Abb. 4) aufgeführt. Unter Flexibilität im Umgang mit mathematischen Situationsstrukturen verstehen wir in diesem Sinne die Fähigkeit, Situationsmodelle mathematischer Strukturen (z. B. hier additive Situationsstrukturen) durch Inferenzen mit zusätzlichen strukturellen Eigenschaften anzureichern. Dies umfasst, eine verbal beschriebene Situation auf der Ebene der Situationsstruktur unterschiedlich deuten zu können, nicht explizit im Aufgabentext enthaltene Aspekte der Situationsstruktur durch Inferenzen beim Lesen für das Lösen von Textaufgaben heranziehen zu können und verschiedene verbale Beschreibungen daraufhin prüfen zu können, ob sie zur gleichen Situationsstruktur passen oder einander widersprechen. In Anlehnung an Greeno (1980) wäre ein Beispiel dafür, einen als Text beschriebenen Vergleich zweier Mengen auch als dynamische Situation (z. B. Ausgleich) deuten zu können. Ein anderes Beispiel wäre, verschiedene Beschreibungen eines quantitativen Vergleichs als gleich bzw. widersprüchlich zu erkennen oder selbstständig ineinander überführen zu können (Stern 1993).

Eine besondere Rolle sollte diese Flexibilität dann spielen, wenn individuelle Situationsmodelle, die auf Basis der sprachlichen Realisierung initial rekonstruiert wurden, durch weitere Inferenzen auf der Ebene der Situationsstrukturen angereichert werden. Eine geringe Flexibilität im Umgang mit Situationsstrukturen könnte eine Ursache für die Unterschiede zwischen Textaufgaben sein, die verschiedene Aspekte der Situationsstruktur (d.h. Formulierungsrichtung, semantische Struktur, gesuchte Menge) sprachlich präsentieren. Den oben beschriebenen Förderansätzen liegt die Hypothese zugrunde, dass eine Intervention zur Flexibilität im Umgang mit mathematischen Situationsstrukturen eine Möglichkeit bietet, Lernende beim Lösen von Textaufgaben zu unterstützen. Auch praxisorientierte Beiträge aus der Mathematikdidaktik schlagen ähnliche Ansätze vor (Fromme et al. 2011). Arbeiten, die eine solche Hypothese prüfen bzw. die Wirksamkeit einer solchen Intervention untersuchen, sind uns nicht bekannt.

Die bisherigen Ergebnisse zu Schwierigkeitsunterschieden zwischen Textaufgaben, die unterschiedliche Situationsstrukturen enthalten (Stern 1998; z. B. Riley und Greeno 1988), deuten zumindest an, dass Lernende eine solche Umdeutung beim Lösen von Textaufgaben nicht völlig spontan oder problemlos anwenden.

Als Ursache dafür kommt einerseits in Frage, dass Lernende nicht über die beschriebene Flexibilität im Umgang mit Situationsstrukturen verfügen. Andererseits wäre auch denkbar, dass Lernende diese Strategie allgemein oder in bestimmten Situationen nicht anwenden, obwohl sie über das notwendige Wissen verfügen. Beispielsweise wäre es möglich, dass Lernende die Strategie nur dann spontan anwenden, wenn schon ein alternatives Situationsmodell aufgebaut und einer mathe- 
matischen Struktur zugeordnet wurde. In diesem Fall könnte das Lösen aufeinanderfolgender, strukturell ähnlicher Aufgaben die Anwendung der Strategie anregen. Im Sinne des Rahmenmodells in Abb. 1 meinen wir mit strukturell ähnlich hier, dass die eine Situation (z. B. eine Vergleichssituation) durch Inferenzen auf der Ebene der Situationsstrukturen in die andere Situation (z.B. eine Ausgleichssituation) umgedeutet werden kann. Flexibilität im Umgang mit Situationsstrukturen und deren strategische Nutzung könnte sich dabei so äußern, dass Lernende strukturelle Ähnlichkeiten nutzen, um das Lösen einer schwierigeren Aufgabe auf eine einfachere, vorhergehende Aufgabe zurückzuführen. Eine solche strategische Nutzung sollte schwierigere Aufgabentypen (z. B. Vergleichsaufgaben) vor allem dann vereinfachen, wenn die Situationsstruktur der vorherigen, einfacheren Aufgabe durch wenige Inferenzen so ergänzt werden kann, dass sie zur dargebotenen sprachlichen Realisierung der folgenden, schwierigeren Textaufgabe passt. In diesem Fall ist es nicht mehr notwendig, ein vollständig neues Situationsmodell aufzubauen. Es reicht prinzipiell aus, den Text der zweiten Aufgabe mit dem bereits aufgebauten Situationsmodell aus der vorherigen Aufgabe durch Inferenzen auf der Ebene der Situationsstrukturen mental abzugleichen. So könnten Lernende erkennen, dass das Situationsmodell der vorherigen Aufgabe und damit auch die dazu identifizierte mathematische Struktur erneut verwendet werden kann.

Eine andere Ursache für fehlende Strategieanwendung könnten sogenannte soziomathematische Normen sein, also informelle, in der Unterrichtsinteraktion sozial konstruierte Normen dazu, wie Aufgaben im Mathematikunterricht anzugehen sind (z. B. Yackel und Cobb 1996). So könnten Lernende davon ausgehen, dass mathematische Lösungsansätze immer direkt aus der gestellten Aufgabe ohne weitere Elaboration des Situationsmodells generiert werden können. Eine solche Norm würde auch die häufig dokumentierte oberflächliche Verarbeitung von Textaufgaben erklären (Verschaffel et al. 1994). Sollte ein einfacher Hinweis auf die Möglichkeit der Anwendung einer Umdeutungsstrategie ausreichen, um die Leistung der Lernenden deutlich zu verbessern, so würde das für die zweite Erklärung sprechen - und die Notwendigkeit einer Trainingsstudie zur Flexibilität in Frage stellen.

Das beschriebene Konzept der Flexibilität im Umgang mit mathematischen Situationsstrukturen klingt implizit in den Arbeiten von Greeno (1980) und Stern (1993) an. Wir schlagen nun vor, diese Flexibilität als individuelles Fähigkeitskonstrukt zu verstehen, und dieses auf Leistungen der Lernenden, Förderbarkeit und Relevanz für die Leistung bei Textaufgaben zu untersuchen. Ziel des vorliegenden Beitrags ist in diesem Sinne die Untersuchung der vorliegenden Flexibilität von Lernenden.

\section{Eine Studie zur Flexibilität im Umgang mit mathematischen Situationsstrukturen}

\subsection{Ziele und Fragestellungen}

Anhand des vorgestellten Modells (Abb. 1) lassen sich die wesentlichen Ziele eines Förderkonzepts zum Umgang mit schwierigen Textaufgaben illustrieren, welches die verschiedenen Umdeutungsmöglichkeiten auf Sprach- und Situationsebene in 
den Blick nimmt und explizit Umdeutungsstrategien vermittelt. Dabei handelt es sich um zwei grundlegende Strategietypen, welche in Abb. 1 mit Pfeilen auf der Ebene der Situationsstruktur beispielhaft gekennzeichnet wurden. Einerseits kann in manchen Fällen die semantische Struktur einer Textaufgabe in eine andere semantische Struktur umgedeutet werden (exemplarisch (4) in Abb. 1). Beispielsweise könnte die Vergleichssituation „Anna hat 3 Kekse. Ben hat 5 Kekse. Ben hat 2 Kekse mehr als Anna.“ auch als Ausgleich formuliert werden: „Wenn Ben 2 Kekse isst, hat er genauso viele wie Anna.“ Andererseits könnte aber in derselben Vergleichssituation auch die Formulierungsrichtung umgedeutet werden: „Anna hat 2 Kekse weniger als Ben." (exemplarisch (5) in Abb. 1). Durch diese Umdeutungen wird die Situationsstruktur verändert. In letzterem Fall wird die schwierigere Vergleichsaufgabe mit gesuchter Ausgangsmenge zu einer einfacheren Vergleichsaufgabe mit gesuchter Ergebnismenge.

Vor einer Erprobung eines solchen Förderkonzepts stellen sich jedoch, wie im Forschungsstand ausgeführt, noch offene Fragen, denen im Rahmen der hier vorgestellten Studie nachgegangen wird. Das erste Ziel der Studie war es, wesentliche Ergebnisse des Forschungsstandes zu prüfen, die dem Förderkonzept zugrunde liegen. Dies betraf unter anderem die Frage, welche Merkmale der im Aufgabentext beschriebenen Situation die Schwierigkeit von Textaufgaben beeinflussen.

Fragenkomplex 1 Welche der Aufgabenmerkmale semantische Struktur, Formulierungsrichtung und gesuchte Menge erklären Schwierigkeitsunterschiede zwischen additiven Textaufgaben?

Basierend auf bisherigen Ergebnissen erwarteten wir, dass Vergleichsaufgaben schwieriger ausfallen würden als Ausgleichsaufgaben (H1.1) und Veränderungsaufgaben (H1.2). Aufgrund von Schwierigkeiten der Lernenden in Bezug auf die Symmetrie des quantitativen Vergleichs (s. oben) sollten sich höhere Lösungsraten für Aufgaben zeigen, bei denen die Formulierungsrichtung mit der zu wählenden Rechenoperation konsistent ist (z. B. aufsteigende Formulierung ,mehr als“, „,dazubekommen“ und „Addition der beiden gegebenen Größen“ als korrekte Rechenoperation; H1.3). In bisherigen Studien wurden weitere Merkmale (ausgetauschte Objekte, Namen, Zahlenmaterial) der Aufgaben i.d.R. gemeinsam mit den genannten Aufgabenmerkmalen variiert. Ziel dieser Studie war es, die durch diese Merkmale verursachte Variation der Schwierigkeit zu kontrollieren, wobei lediglich kleine Unterschiede erwartet wurden (H1.4).

Als zweites Ziel sollte die Studie untersuchen, ob und ggf. in welchem Umfang Lernende die für das Förderkonzept vorgesehene Strategie der Umdeutung von Situationsstrukturen bereits spontan nutzen bzw. bereits aufgrund eines einfachen Hinweises anwenden können.

Fragenkomplex 2 Inwiefern nutzen Lernende Ähnlichkeiten in den Situationsstrukturen spontan, um Textaufgaben zu lösen? Inwiefern kann eine solche Strategie bereits durch einen einfachen Hinweis im Rahmen der Testsituation angeregt werden?

Eine solche Nutzung sollte sich darin äußern, dass sich für Textaufgaben höhere Lösungsraten zeigen, wenn direkt vorher eine Aufgabe gelöst wurde, der eine 
strukturell ähnliche Situation zugrunde lag (H2.1). Aufgrund der Ergebnisse zu Schwierigkeitsunterschieden fokussierten wir uns in dieser Studie auf Situationen, denen unterschiedliche semantische Strukturen zugrunde lagen. Wir hatten keine Hypothesen dazu, in welchem Umfang sich eine solche Erleichterung aufgrund vorhergehender Aufgaben mit ähnlichen Situationen zeigen würde. Wenn Lernende entsprechende Umdeutungsstrategien nutzen, erwarteten wir jedoch größere Effekte, wenn Vergleichsaufgaben in (leichtere) dynamische Strukturen umgedeutet werden, als anders herum (H2.2). Für den Fall, dass Lernende zwar über das notwendige Wissen zur Umdeutung verfügen, dieses jedoch beim Lösen von Aufgaben lediglich nicht oder nur eingeschränkt anwenden, erwarteten wir, dass die zu H2.1 und H2.2 genannten Effekte ausgeprägter ausfallen würden, wenn direkt vor der Aufgabenbearbeitung ein expliziter Hinweis gegeben wird, der die Nutzung der strukturellen Ähnlichkeit zwischen den Aufgaben nahelegt (H2.3).

\subsection{Methode}

Zur Bearbeitung dieser Fragen wurde eine Querschnittstudie mit Paper-Pencil-Tests in acht Klassen der Jahrgangsstufe 2 drei verschiedener Grundschulen $(N=139)$ etwa zur Mitte des Schuljahres durchgeführt, wobei mit $48 \%$ weiblichen Probanden die Geschlechterverteilung in etwa ausgeglichen war. Zum Zeitpunkt der Erhebung waren die teilnehmenden Schülerinnen und Schüler im Schnitt 7,56 Jahre alt. Bezüglich der Familiensprache wurden Selbstberichte der Kinder erhoben, nach denen $40 \%$ der Kinder zu Hause nur deutsch sprechen, $43 \%$ der Kinder zu Hause deutsch und eine andere Sprache sprechen und 16\% der Kinder zu Hause nicht deutsch sprechen (ein fehlender Wert).

\subsubsection{Materialien}

Jede Schülerin und jeder Schüler bearbeitete vierundzwanzig Aufgaben aus einem größeren Pool von additiven Textaufgaben. Zur Untersuchung von Fragenkomplex 2 wurden die Textaufgaben paarweise eingesetzt (Textaufgaben-Paare). Beiden Aufgaben eines solchen Textaufgaben-Paares lag jeweils derselbe Kontext (Namen, Objekte) und dieselbe mathematische Struktur zugrunde, welche jedoch mittels unterschiedlicher semantischer Strukturen umgesetzt wurde. Aufgaben eines Textaufgaben-Paares stellten also eine ähnliche, aber doch verschiedene Situation dar. Ein beispielhaftes Textaufgaben-Paar bestand aus Aufgabe 1: „Nils hat 13 Kastanien, Maria hat 8 Kastanien. Wie viele Kastanien muss Nils noch abgeben, damit er gleich viele wie Maria hat?" und Aufgabe 2: „Nils hat 13 Kastanien, Maria hat 8 Kastanien. Wie viele Kastanien hat Maria weniger als Nils?". Beide Aufgaben enthalten dieselbe mathematische Struktur (13-8=5), denselben Kontext (Nils und Maria als Personen, Kastanien als Objekte), dieselbe gesuchte Menge (Veränderungsmenge) und dieselbe Formulierungsrichtung (absteigend). Der einzige Unterschied ist die jeweils enthaltene semantische Struktur. Während die erste Aufgabe einen Ausgleich darstellt, handelt es sich bei der zweiten Aufgabe um einen Vergleich. Analog wurden Paare aus Vergleichs- und Veränderungsaufgaben konzipiert. Die sprachliche Realisierung der unterschiedlichen Situationsstrukturen wurde bzgl. 
der verwendeten Sprachregister so weit wie möglich kontrolliert, indem Wortschatz und grammatikalische Strukturen möglichst wenig variiert wurden.

Grundlage des Designs war die Annahme, dass insbesondere eine Umdeutung der semantischen Struktur „Vergleich“ in „Veränderung“ bzw. „Ausgleich“ für Lernende hilfreich sein könnte, da sich diese beiden semantischen Strukturen in bisherigen Studien als einfacher herausstellten (Stern 1994) und in Modellen des Zahlbegriffserwerbs früheren Phasen der Entwicklung zugeordnet werden als Vergleichsaufgaben (Fritz et al. 2018). Als besonders hilfreich wurde die Umdeutung von Vergleichsaufgaben in Ausgleichsaufgaben eingeschätzt, da diese zusätzlich zu den aufgeführten Gründen genau wie Vergleichsaufgaben disjunkte Mengen enthalten, und so die Umdeutung leichter fallen könnte. Vereinigungsaufgaben wurden nicht berücksichtigt, da sie sich im Rahmen von Simplexaufgaben schwer mit Vergleichsaufgaben kombinieren lassen, wie sich auch schon in einer Examensarbeit (Weber 2016) zeigte. Für die Zusammenstellung aller möglichen Versionen von Textaufgaben-Paaren wurden Kombinationen aus den semantischen Strukturen im Paar (Vergleich und Ausgleich, Vergleich und Veränderung), der Formulierungsrichtung (aufsteigend, absteigend) und der gesuchten Menge (Ausgangs-, Veränderungs- und Ergebnismenge) systematisch variiert. Dadurch entstanden zwölf prototypische Textaufgaben-Paare, die im Anschluss in zwölf verschiedene Kontexte, bestehend aus unterschiedlichen beteiligten Personen, Objekten und Zahlenmaterial, eingebettet wurden. Schließlich wurde noch ein zweiter Satz an Textaufgaben-Paaren generiert, indem die Reihenfolge der beiden Aufgaben im Paar umgekehrt wurde. Tab. 2 (Anhang) zeigt Beispiele für alle in der Studie eingesetzten Typen von Textaufgaben.

\subsubsection{Ablauf}

Die Erhebung fand als Paper-Pencil-Test im Klassenverband statt. Jedes Kind bearbeitete zwölf unterschiedliche, zufällig ausgewählte Textaufgaben-Paare in zufälliger Reihenfolge. Die Paare an zweiter und an siebter Stelle wurden durch DistraktorenPaare ersetzt, bei welchen jeweils die zweite Textaufgabe im Paar so verändert wurde, dass die dazugehörige Rechenoperation genau umgekehrt im Vergleich zur ersten Textaufgabe war. So sollte vermieden werden, dass die Lernenden grundsätzlich die Rechnung der ersten Aufgabe im Textaufgaben-Paar für die zweite Aufgabe übernehmen, ohne diese wirklich zu lesen. Das Testheft war so strukturiert, dass pro Seite nur eine Textaufgabe zu bearbeiten war. Mit der Anweisung, nicht zurückzublättern, wurde eine nachträgliche Korrektur von Aufgabenbearbeitungen durch die Lernenden so weit wie möglich vermieden. Zur Bearbeitung der Textaufgaben-Paare wurde den Lernenden 40 min Zeit gegeben. Nach der Hälfte der Bearbeitungszeit wurde eine kurze Pause vorgenommen.

Bei der Hälfte der teilnehmenden Klassen wurde zu Beginn ein Hinweis gegeben, der die Nutzung der ersten Textaufgabe im Paar für die Bearbeitung der zweiten unterstützen sollte. In diesen Klassen erklärte die Person, die die Erhebung moderierte, vor Beginn der Arbeit an den Textaufgaben-Paaren, dass manche Aufgaben sehr ähnlich zueinander sind. Die Schülerinnen und Schüler wurden darauf hingewiesen, dass es in manchen der Textaufgaben um genau dasselbe gehen würde, wie in der Aufgabe davor - nur mit anderen Worten beschrieben. Für diesen Fall wurden 
die Lernenden ermutigt, die Aufgabe nicht erneut zu rechnen, sondern die Rechnung aus der vorhergehenden Aufgabe zu übertragen. Anhand dieser experimentellen Manipulation sollte kontrolliert werden, ob lediglich soziomathematische Normen bzw. Überzeugungen der Lernenden zum in der Situation erwarteten Problemlöseprozess Ursache für eine fehlende Nutzung von vorhandenen Fähigkeiten zur Umdeutung von Situationsstrukturen sind.

\subsubsection{Kodierung}

Aus jeder Lösung wurden zwei Leistungsmaße kodiert. Beim ersten Maß (korrektes Ergebnis) wurde eine Antwort nur als korrekt gewertet, wenn das berechnete numerische Ergebnis mit dem richtigen Ergebnis übereinstimmte. Beim zweiten Maß (korrekte Rechenoperation) wurde eine Antwort akzeptiert, wenn mindestens die Rechnung oder das Ergebnis richtig war. Dabei wurden alle Rechnungen und Rechenstrategien, die mathematisch äquivalent zu einer zur Aufgabe passenden mathematischen Struktur (siehe Abb. 1) sind, als korrekte Rechenoperation eingestuft. Dazu gehören z. B. das schrittweise Rechnen bis zum Zehner $(7+8=7+3+5=15)$, aber auch Tausch-, Umkehr- und Ergänzungsstrategien.

\subsubsection{Statistische Analyse}

Zur inferenzstatistischen Auswertung wurden verallgemeinerte lineare Mischmodelle für dichotome Daten mit einer logistischen Linkfunktion (Bates et al. 2014) verwendet, in denen die Korrektheit der Antwort für jede beantwortete Aufgabe getrennt durch Personen- und Aufgabenmerkmale vorhergesagt wurde. Zusätzlich wurden in allen Modellen die Abhängigkeiten zwischen Antworten derselben Person mittels eines entsprechenden Zufallsfaktors berücksichtigt. Abhängigkeiten zwischen Antworten auf Textaufgaben zum gleichen Kontext sowie Antworten von Lernenden derselben Klasse wurden mit entsprechenden Zufallsfaktoren modelliert, wenn sie einen identifizierbar von Null verschiedenen Anteil der Varianz erklärten. Zur Untersuchung von Haupt- und Interaktionseffekten der Aufgabenmerkmale wurden Likelihood-Ratio-Tests auf Basis der Chi-Quadrat-Statistik herangezogen. Dieser Test vergleicht die Passung des Modells mit dem jeweiligen Effekt mit der Passung des Modells ohne den Effekt. Zum Vergleich von Lösungsraten zwischen verschiedenen Bedingungen wurden weiter geplante Kontraste der jeweiligen geschätzten Randmittel berechnet ${ }^{3}$. Die berichteten nicht-standardisierten Regressionskoeffizienten sind als Differenzwerte auf einer Log-Odds-Ratio-Skala ähnlich Differenzen von Itemparametern in einem IRT-Modell zu interpretieren. Alle Berechnungen wurden in R mit den Paketen lme4 (Bates et al. 2014) und emmeans (Lenth et al. 2018) durchgeführt.

\footnotetext{
${ }^{3}$ Bei mehrfacher Testung von Kontrasten zu einem Effekt wurde nach Bonferroni adjustiert.
} 


\section{Ergebnisse}

\subsection{Schwierigkeitsunterschiede durch semantische Strukturen, Formulierungsrichtung und gesuchte Menge}

Um den ersten Fragenkomplex zu bearbeiten, wurden nur die jeweils ersten Aufgaben aus den Textaufgaben-Paaren analysiert. Die Distraktorpaare blieben dabei unberücksichtigt. Insgesamt lagen bei diesen Aufgaben von 1390 vorgelegten Textaufgaben-Paaren 1288 auswertbare Antworten vor, von denen 941 (73,1\%) numerisch korrekt waren und bei 1020 (79,2\%) die korrekte Operation gewählt wurde. In allen Auswertungen zeigten sich kaum identifizierbare Unterschiede der Häufigkeiten korrekter Ergebnisse bzw. korrekter Rechenoperationen zwischen den verschiedenen Kontexten. Der dafür vorgesehene Zufallseffekt erklärte jeweils wie erwartet (H1.4) keinen identifizierbaren oder nur einen sehr kleinen Varianzanteil (weniger als $0,01 \%$ ).

In einem ersten Schritt wurde nur der Einfluss der Haupteffekte der in den Textaufgaben umgesetzten semantischen Struktur, der gesuchten Menge und der Formulierungsrichtung analysiert.

\subsubsection{Semantische Struktur}

Es zeigten sich keine signifikanten Unterschiede zwischen den semantischen Strukturen in Bezug auf die Häufigkeit numerisch korrekter Ergebnisse (Veränderung: 77,1\%; Ausgleich: 71,0\%; Vergleich: 72,1\%; LR-Test: $\left.\chi^{2}(2)=4,06 ; p=0,13\right)$. Bei Ausgleichsaufgaben wurde jedoch signifikant seltener die korrekte Operation identifiziert $(\mathrm{B}=0,60 ; p=0,03)$ als bei Veränderungsaufgaben (Veränderung: $82,4 \%$; Ausgleich: 75,4\%; Vergleich: 76,3\%; LR-Test: $\left.\chi^{2}(2)=6,71 ; p=0,027\right)$. Insbesondere konnten H1.1 und H1.2 zur besonderen Schwierigkeit von Vergleichsaufgaben nicht bestätigt werden.

\subsubsection{Formulierungsrichtung}

Der Haupteffekt der Formulierungsrichtung auf die Häufigkeit korrekter Ergebnisse (LR-Test: $\left.\chi^{2}(1)=0,54 ; p=0,46\right)$ und die Häufigkeit korrekt identifizierter Rechenoperationen (LR-Test: $\left.\chi^{2}(1)=2,70 ; p=0,10\right)$ war jeweils nicht signifikant.

\subsubsection{Gesuchte Menge}

Es zeigten sich signifikante Unterschiede für die Häufigkeit korrekter Ergebnisse (LR-Test: $\left.\chi^{2}(2)=20,99 ; p<0,001\right)$ und korrekter Rechenoperationen (LR-Test: $\left.\chi^{2}(2)=32,72 ; p<0,001\right)$ nach der gesuchten Menge. Bei Aufgaben mit gesuchter Ergebnis- $(78,1 \%$ korrekte Ergebnisse; $\mathrm{B}=0,51 ; p<0,001)$ oder Veränderungsmenge $(74,0 \% ; \mathrm{B}=0,81 ; p=0,01)$ wurde signifikant häufiger das korrekte Ergebnis angegeben als bei Aufgaben mit gesuchter Ausgangsmenge (66,8\%). Der Unterschied zwischen Aufgaben mit gesuchter Ergebnis- und Veränderungsmenge war nicht signifikant $(B=0,30 ; p=0,22)$. Korrekte Rechenoperationen wurden häufiger 
Abb. 2 Überblick über die Lösungsraten (a korrekte Ergebnisse, b korrekte Operationen) nach gesuchter Menge (A Ausgangsmenge, $\mathrm{V}$ Veränderungsmenge, E Ergebnismenge) und Formulierungsrichtung
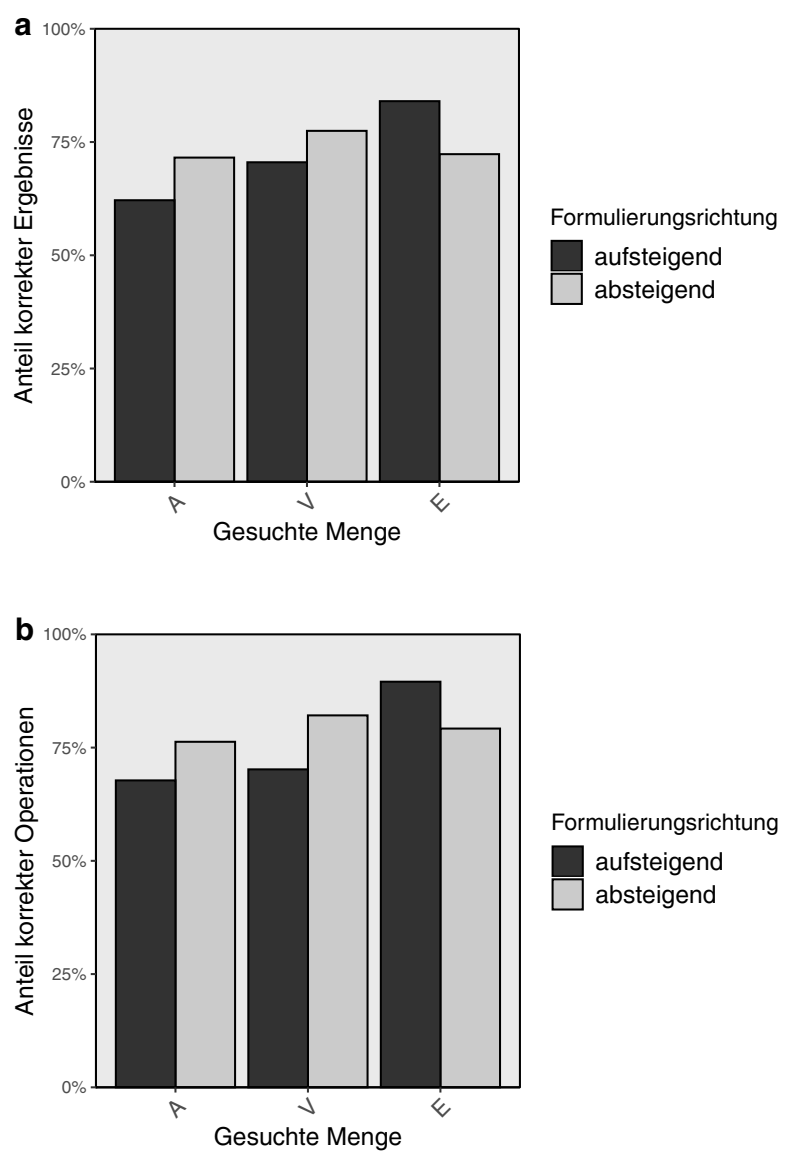

bei Aufgaben mit gesuchter Ergebnismenge ( $84,3 \%$ korrekte Rechenoperationen) als bei Aufgaben mit gesuchter Veränderungs- $(76,3 \%$; $\mathrm{B}=0,78 ; p<0,001)$ oder Ausgangsmenge $(72,0 \% ; \mathrm{B}=1,15 ; p<0,001)$ identifiziert. Der Unterschied zwischen Aufgaben mit gesuchter Ausgangs- und Veränderungsmenge war nicht signifikant $(\mathrm{B}=0,37 ; p=0,14)$. Besonders Aufgaben mit gesuchter Ausgangsmenge erwiesen sich also als schwieriger als solche mit gesuchter Ergebnismenge. Dies deckt sich mit den älteren Befunden von Stern (1993).

\subsubsection{Interaktionseffekte}

In einem zweiten Schritt wurden die Interaktionen zwischen den drei Haupteffekten analysiert. Es zeigte sich sowohl für die Häufigkeit korrekter Ergebnisse (LR-Test: $\left.\chi^{2}(2)=22,40 ; p<0,001\right)$ als auch für die Häufigkeit korrekter Operationen (LR-Test: $\left.\chi^{2}(2)=30,84 ; p<0,001\right)$ ein signifikanter Interaktionseffekt von gesuchter Menge und Formulierungsrichtung. Für die Häufigkeit korrekter Operationen zeigte sich weiterhin eine Interaktion von semantischer Struktur und Formulierungsrichtung 
(LR-Test: $\left.\chi^{2}(2)=8,20 ; p=0,017\right)$. Die Dreifachinteraktion war für beide Leistungsmaße nicht signifikant (Ergebnisse: LR-Test: $\chi^{2}(4)=2,75 ; p=0,60$; Operationen: LR-Test: $\left.\chi^{2}(4)=2,61 ; p=0,62\right)$.

\subsubsection{Gesuchte Menge und Formulierungsrichtung}

Abb. 2 zeigt die Anteile korrekter Ergebnisse (Abb. 2a) bzw. korrekter Rechenoperationen (Abb. 2b) nach gesuchter Menge und Formulierungsrichtung. Wie erwartet (H1.3) waren richtige Ergebnisse und richtige Operationen häufiger bei Aufgaben, in denen die Formulierungsrichtung mit der Rechenoperation übereinstimmte (gesuchte Ergebnismenge bzw. absteigende Formulierung bei gesuchter Veränderungsmenge: 77,9\% korrekte Ergebnisse; 83,6\% korrekte Operationen) als bei Aufgaben, bei denen die Formulierung nicht mit der Rechenoperation übereinstimmte $(68,0 \%$ korrekte Ergebnisse; 71,4\% korrekte Operationen; geplanter Kontrast für Ergebnisse $\mathrm{B}=0,76 ; p<0,001 \mathrm{bzw}$. für Operationen $\mathrm{B}=1,18 ; p<0,001)$.

Abb. 2 weist jedoch auch auf Unterschiede zwischen absteigend und aufsteigend formulierten Aufgaben innerhalb von Aufgaben mit gesuchter Ausgangsmenge bzw. Ergebnismenge hin. Bei einer gesuchten Ausgangsmenge sind die Formulierungsrichtungen der Rechenoperation immer entgegengesetzt, bei gesuchter Ergebnismenge stimmen sie stets überein. Die Übereinstimmung von Formulierungsrichtung und Rechenoperation kann hier also jeweils nicht die Ursache für die Unterschiede in den Lösungsraten sein. In beiden Fällen wurden die Aufgaben häufiger korrekt gelöst, in denen ein additives Modell die Situation korrekt beschreibt. Dies wurde explorativ mit Hilfe geplanter Kontraste untersucht. Bei Aufgaben, für die eine Addition (oder eine äquivalente mathematische Struktur) das korrekte Modell ist (aufsteigend formulierte Aufgaben mit gesuchter Ergebnismenge, absteigend formulierte Aufgaben mit gesuchter Ausgangsmenge), waren korrekte Ergebnisse (77,9\%; B =0,54; $p<0,001)$ bzw. korrekte Rechenoperationen $(82,8 \% ; \mathrm{B}=0,81 ; p<0,001)$ signifikant häufiger als bei Aufgaben, bei denen das korrekte Modell eine Subtraktion (oder eine äquivalente mathematische Struktur) war (70,6\% korrekte Ergebnisse; 74,8\% korrekte Operationen).

\subsubsection{Semantische Struktur und Formulierungsrichtung}

Bezüglich dieser Interaktion in Bezug auf die Identifikation der korrekten Rechenoperation zeigten die einzelnen Kontraste zwischen Aufgaben mit aufsteigender und absteigender Formulierung keine signifikanten Unterschiede. Deskriptiv wurden jedoch bei aufsteigend formulierten Veränderungs- $(80,1 \%)$ und Vergleichsaufgaben $(73,0 \%)$ die korrekte Operation seltener identifiziert als bei entsprechenden absteigenden Aufgaben (84,7\% bzw. 79,7\%). Bei Ausgleichsaufgaben zeigte sich die umgekehrte Tendenz (aufsteigend: 77,9\%; absteigend: 72,9\%).

\subsection{Nutzung von Ähnlichkeiten zwischen Aufgaben innerhalb eines Paares}

Mit dem zweiten Fragenkomplex soll ergründet werden, inwiefern Lernende ähnliche Situationsstrukturen bei der Lösung von Textaufgaben bereits spontan bzw. 
aufgrund eines einfachen Hinweises nutzen. Um dies zu analysieren, wurden die zweiten Aufgaben der Textaufgaben-Paare zusätzlich herangezogen und getrennt nach den Paaren mit den semantischen Strukturen Veränderung und Vergleich bzw. Ausgleich und Vergleich analysiert. Die Haupteffekte für die semantische Struktur ergaben dasselbe Effektmuster wie in den Analysen zum ersten Fragenkomplex.

\subsubsection{Aufgabenposition}

Unseren Annahmen entsprechend analysierten wir zunächst die Effekte der Aufgabenposition im Textaufgaben-Paar (erste vs. zweite Aufgabe). Diese Haupteffekte waren in allen Fällen nicht signifikant (z. B. LR-Test für korrekte Ergebnisse bei Paaren aus Vergleichs- und Veränderungsaufgaben: Korrekte Ergebnisse $\chi^{2}(1)=1,61$; $p=0,20$; korrekte Operationen $\left.\chi^{2}(1)=1,67 ; p=0,28\right)$. Die Hypothese, dass eine vorher bearbeitete Aufgabe das Lösen einer folgenden Aufgabe ähnlicher Situationsstruktur unterstützt, konnte also nicht bestätigt werden (H2.1).

\subsubsection{Aufgabenposition und semantische Struktur}

Die Interaktion aus Aufgabenposition und semantischer Struktur war in allen Fällen nicht signifikant (z.B. LR-Test für korrekte Ergebnisse bei Paaren aus Vergleichsund Veränderungsaufgaben: Korrekte Ergebnisse $\chi^{2}(1)=0,13 ; p=0,72$; korrekte Operationen $\left.\chi^{2}(1)=0,58 ; p=0,81\right)$. Insgesamt konnte also die Annahme nicht bestätigt werden, dass insbesondere Vergleichsaufgaben besser gelöst wurden, wenn direkt davor eine strukturell ähnliche Veränderungs- oder Ausgleichsaufgabe bearbeitet wurde (H2.2).

\subsubsection{Hinweis auf Strukturgleichheit}

Im nächsten Schritt wurde der Effekt des Hinweises auf die ähnliche Situationsstruktur in die Modelle aufgenommen. Dieser Effekt und seine Interaktion mit der Aufgabenposition waren weder für die Paare aus Veränderungs- und Vergleichsaufgaben (z. B. LR-Test für korrekte Ergebnisse: Hinweis $\chi^{2}(1)=0,97 ; p=0,32$; Interaktion $\left.\chi^{2}(1)=0,11 ; p=0,73\right)$, noch für die Paare aus Ausgleichs- und Vergleichsaufgaben signifikant. Ebenso waren die Interaktionen von Hinweis und semantischer Struktur sowie die Dreifachinteraktion aus Hinweis, Aufgabenposition und semantischer Struktur nicht signifikant. Insgesamt zeigte also auch der in einem Teil der Klassen gegebene Hinweis keine Auswirkungen auf die Nutzung der strukturellen Ähnlichkeit der Textaufgaben in einem Aufgabenpaar (H2.3).

\subsubsection{Weiterführende Analysen}

Bereits die Ergebnisse zum ersten Fragenkomplex legen nahe, dass für unsere Stichprobe nicht wie angenommen die jeweilige semantische Struktur Unterschiede in der Schwierigkeit der Textaufgaben erklärt, sondern besonders die Formulierungsrichtung der Aufgabe in Kombination mit der in der Aufgabe gesuchten Menge. Explorativ wurde deshalb weiter untersucht, ob sich Interaktionseffekte der Formu- 
lierungsrichtung bzw. gesuchten Menge mit Aufgabenposition und Hinweisen auf die Strukturgleichheit für einzelne Aufgabentypen nachweisen lassen. Es zeigte sich lediglich bei Paaren aus Ausgleichs- und Vergleichsaufgaben ein Interaktionseffekt aus gesuchter Menge und Aufgabenposition (LR-Test: $\chi^{2}(2)=6,18 ; p<0,05$ ): Die korrekte Operation wurde hier bei Aufgaben mit gesuchter Veränderungsmenge signifikant häufiger identifiziert, wenn die Aufgabe an zweiter Stelle im Paar stand $(81,3 \%)$, als wenn es sich um die erste der beiden Aufgaben in einem Paar handelte (76,1\%; $\mathrm{B}=0,38 ; p<0,05)$. Die vorliegenden Daten lieferten keine Hinweise darauf, dass dieser Unterschied unter bestimmten Umständen besonders ausgeprägt gewesen wäre, z. B. wenn die Vergleichsaufgabe an zweiter Position im Paar stand oder wenn ein Hinweis zur Strukturgleichheit der Aufgaben gegeben wurde. Das Effektmuster könnte darauf hindeuten, dass Schülerinnen und Schüler die strukturelle Ähnlichkeit zwischen Vergleichs- und Ausgleichsaufgaben zu einem gewissen Grad nutzen, um mathematische Modelle aufzustellen. Dieser Interaktionseffekt aus gesuchter Menge und Aufgabenposition war jedoch nicht signifikant, wenn korrekte Ergebnisse als Kriteriumsvariable genutzt wurden (LR-Test: $\left.\chi^{2}(2)=1,91 ; p=0,38\right)$. Es fanden sich keine Hinweise darauf, dass die Lernenden mehr von Ausgleichsaufgaben für das spätere Lösen von Vergleichsaufgaben profitierten oder umgekehrt.

\section{Diskussion}

\subsection{Schwierigkeitsunterschiede zwischen Textaufgaben}

Ein erstes Ziel des Beitrags war es zunächst, systematisch zu untersuchen, welche der in der Literatur berichteten Faktoren die Schwierigkeit von Textaufgaben in unserer Zielpopulation beeinflussen. Dafür wurden nur die jeweils ersten Aufgaben jedes Textaufgaben-Paares herangezogen. Insgesamt erwiesen sich die Unterschiede zwischen den Aufgabentypen als gering, die Lösungsraten der verschiedenen Aufgabentypen fielen im Vergleich zu älteren Studien (z. B. Stern 1994 in Jahrgangsstufe 1) homogener und etwas höher aus. Oberflächenmerkmale wie die in der Aufgabe verwendeten Objekte (Murmeln, Blumen ...) und das konkret gewählte Zahlenmaterial hatten kaum beobachtbare Auswirkungen auf die Aufgabenschwierigkeit. Darüber hinaus zeigten sich nur geringe Unterschiede nach der in den Textaufgaben präsentierten semantischen Struktur. Dies steht im Kontrast zu älteren Ergebnissen von z. B. Stern (1998) in Jahrgangsstufe 1, die Vergleichsaufgaben als besonders schwierig identifiziert hatten. Es scheint den untersuchten Lernenden der Jahrgangsstufe 2 vergleichsweise leicht zu fallen, die drei untersuchten semantischen Strukturen aus der sprachlichen Realisierung zu rekonstruieren und mit einer mathematischen Struktur zu beschreiben. Eine Erklärung könnte darin liegen, dass unsere Lernenden Mitte der zweiten Jahrgangsstufe bereits substantielle Erfahrungen zu allen semantischen Strukturen gesammelt haben, andererseits aber auch von einem seit den vorhandenen Studien veränderten Lernangebot, z.B. aufgrund einer verstärkten Behandlung von unterschiedlichen Typen realitätsnaher Aufgabenstellungen im Unterricht, profitieren. Besonders für Vergleichsaufgaben erscheint Letzteres durchaus plausibel, da sie in neueren Modellen des Zahlbegriffserwerbs eine zentrale Rolle einnehmen 
(z.B. Krajewski und Schneider 2009). Die deskriptiven Ergebnisse von Fritz et al. (2018) legen weiterhin nahe, dass zu Beginn der Jahrgangsstufe 2 bereits ca. ein Viertel der Lernenden über Wissen zum Relationszahlaspekt verfügt, zur Mitte des Schuljahres dürften es mehr Kinder sein. Die im Vergleich mit früheren Studien eher geringen Lösungsraten bei Ausgleichsaufgaben könnten möglicherweise auf Missverständnisse, die aus der Aufgabenformulierung hervorgehen, zurückgeführt werden. In zukünftigen Studien müssten Textaufgaben zum Ausgleich von Mengen noch eindeutiger formuliert werden, sodass ein Ausgleich zwischen den beteiligten Personen (,Wie viel muss Hans von Maria bekommen, damit sie gleich viele haben?") als Interpretation ausgeschlossen wird. Die Antworten der an der Studie teilnehmenden Schülerinnen und Schüler deuten allerdings nicht auf eine solche Fehlinterpretation hin.

Als wesentlich bedeutsamer für die Identifikation der korrekten Rechenoperation und die Berechnung des korrekten Ergebnisses erweist sich in unserer Studie, ob Vergleiche und Handlungen im Aufgabentext aufsteigend oder absteigend formuliert sind. Höhere Lösungsraten zeigen sich, wenn diese Formulierungsrichtung zu der für die Aufgabe direkt anwendbaren Rechenoperation passte. Einerseits kann dies darauf hinweisen, dass Lernende Rechenoperationen alleine anhand einer Signalwortstrategie aufgrund der verbalen Formulierung wählen. Andererseits würde ein fehlendes Verständnis der von Stern (1993) angesprochenen Symmetrie in der sprachlichen Beschreibung quantitativer Vergleiche die Probleme der Lernenden beim Aufbau eines Situationsmodells und damit auch das eventuelle Anwenden einer Signalwortstrategie für Vergleichsaufgaben erklären. Dass sich ähnliche Effekte hier für dynamische semantische Strukturen wie Veränderung und Ausgleich zeigen, könnte dafür sprechen, dass weniger die in Modellen des Zahlbegriffserwerbs angesprochene Bedeutung von Zahlen als Relationen (Fritz et al. 2018) ein zentrales Problem für die Lernenden in unserer Studie war, sondern ganz allgemein die Symmetrie quantitativer Vergleiche (Stern 1993) und Veränderungen. Wenn nun viele Lernende zum Zeitpunkt unserer Studie bereits über einen ausgeprägten Relationszahlbegriff verfügen, wäre es angesichts unserer Ergebnisse zur Rolle von Formulierungsrichtung und gesuchter Menge eine offene Frage, ob ein Verständnis der Symmetrie quantitativer Vergleiche und Veränderungen erst später in der Entwicklung, und möglicherweise simultan für statische und dynamische semantische Strukturen auftritt.

Für das initial angedachte Förderkonzept würde dies bedeuten, dass der Schwerpunkt weniger auf die Beziehungen zwischen unterschiedlichen semantischen Strukturen (z. B. Vergleichs- und Ausgleichsaufgaben) zu legen wäre. In den Fokus eines solchen Konzepts sollte besonders die Symmetrie quantitativer Vergleiche (mehr als, weniger als) bzw. Veränderungen (dazubekommen, abgeben) innerhalb der einzelnen semantischen Strukturen rücken. Deskriptiv zeigt die Studie Unterschiede in der Identifikation der korrekten Operation zwischen auf- und absteigend formulierten Aufgaben, die zwischen den semantischen Strukturen variierten. Auch dies spricht dafür, die Symmetrie für alle drei (bzw. vier) semantischen Strukturen gleichermaßen zu thematisieren.

Darüber hinaus zeigen unsere Analysen ein weiteres, unseres Wissens nach in der Literatur bisher nicht beschriebenes Phänomen. Unabhängig von der gewählten 
Formulierungsrichtung, der jeweils gesuchten Menge und der semantischen Struktur scheinen Lernende in unserer Studie Aufgaben, die eine Addition (oder äquivalente mathematische Strukturen) als mathematisches Modell erfordern, besser zu lösen als solche, die eine Subtraktion (oder äquivalente mathematische Strukturen) erfordern. Einerseits zeigte sich dies für die Anzahl korrekter Ergebnisse. Dies könnte zunächst durch unterschiedliche Schwierigkeit der zugrundeliegenden additiven bzw. subtraktiven Modelle erklärt werden, beispielsweise durch abweichende Zählrichtungen bei Zählstrategien zur direkten Subtraktion (nicht jedoch beim Ergänzen). Andererseits sind z. B. bei Benz (2005) nur geringe Unterschiede in den Lösungsraten zu additiven Textaufgaben in Jahrgangsstufe 2 dokumentiert. Darüber hinaus zeigte sich derselbe Effekt auch für die Anzahl der korrekt identifizierten Rechenoperationen ohne Berücksichtigung der numerischen Ergebnisse. In unserer Studie wurde bei Aufgaben, bei denen eine Subtraktion das korrekte Modell ist, in 18,4\% der Antworten ein additives Modell gewählt. Bei 11,6\% der Antworten auf Aufgaben, bei denen eine Addition das korrekte Modell ist, wurde ein subtraktives Modell gewählt. Dies kann, wenn auch sehr schwach, auch als Präferenz für additive Modelle über subtraktive Modelle interpretiert werden. In der Literatur zum proportionalen Schließen werden Präferenzen für additiv-subtraktive bzw. multiplikativ-proportionale Modelle als eine Erklärung dafür diskutiert, dass einige Lernende Textaufgaben zum proportionalen Schließen mit additiv-subtraktiven Modellen bearbeiten, andere Lernende aber Strategien für Proportionalitäten nutzen, um Textaufgaben mit additiven Situationsstrukturen zu lösen (Modestou und Gagatsis 2013; Resnick und Singer 1993; Van Dooren et al. 2010). Es erscheint plausibel, dass Lernende Operationen nach einer solchen Präferenz wählen, sofern sie auf der Basis ihres Verständnisses der jeweiligen Situation keine klare Entscheidung für eine bestimmte Operation treffen können. Wenn sich eine solche Präferenz in unserem Kontext zugunsten der Addition zeigt, könnte dies an einer wahrgenommen geringeren Schwierigkeit von Additionsaufgaben gegenüber Subtraktionsaufgaben liegen. Auch eine frühere Einführung der Addition im Kontext von Zahlzerlegungen wäre eine mögliche Erklärung für die Präferenz der Addition. Untersuchenswert wäre außerdem, ob eventuell ein unausgewogenes Verhältnis von Additions- und Subtraktionsaufgaben in Schulbüchern bzw. im Unterricht als Ursache in Frage kommt. Unabhängig davon wäre denkbar, dass ein besseres Verständnis der Symmetrie quantitativer Vergleiche bzw. Veränderungen einen solchen Präferenzeffekt verringern könnte.

Zusammenfassend zeigen diese Ergebnisse zum ersten Fragenkomplex der Studie, dass die Lösungsraten in Textaufgaben deutlich davon abhängen, anhand welcher Eigenschaften der Situationsstruktur eine mathematische Struktur in der Textaufgabe dargestellt wird. Es stellt sich also - unabhängig davon, dass einige klassische Ergebnisse für unsere Stichprobe nicht repliziert werden konnten - die Frage, inwiefern eine Anreicherung des Situationsmodells durch alternative Eigenschaften auf der Ebene der Situationsstruktur während des Leseprozesses der Textaufgabe positive Effekte auf den Lösungserfolg haben könnte. 


\subsection{Anreicherung von Situationsmodellen als spontane Lösungsstrategie für Textaufgaben}

In der Literatur finden sich Ansätze, die eine Anreicherung von Situationsstrukturen entweder durch alternative semantische Strukturen (Greeno 1980) oder alternative Sichtweisen auf quantitative Vergleiche und Veränderungen (Stern 1993) als Lösungsstrategie für Textaufgaben vorschlagen. Auch praxisorientierte Beiträge zur Mathematikdidaktik schlagen entsprechende Förderkonzepte vor (Fromme et al. 2011). Der zweite Fragenkomplex beschäftigte sich zur Vorbereitung eines Förderkonzepts damit, ob Lernende die strukturellen Ähnlichkeiten zwischen verschiedenen verbal präsentierten semantischen Strukturen schon ohne Förderung erkennen und für das erfolgreiche Lösen von Textaufgaben nutzen können. Wäre dies der Fall, so müsste ein Förderkonzept weniger darauf abzielen, die Beziehungen zwischen verschiedenen Situationsstrukturen (hier insbesondere zwischen semantischen Strukturen) zu vermitteln, sondern eher darauf, die Nutzung dieses Wissens als Lese- und Problemlösestrategie anzuregen. Aufgrund der unerwartet geringen Schwierigkeitsunterschiede zwischen den semantischen Strukturen in unserer Studie sind die Ergebnisse zu diesem Fragenkomplex nicht erstaunlich. Es zeigen sich kaum Hinweise darauf, dass eine vorausgehende Bearbeitung einer strukturell ähnlichen, jedoch anhand anderer struktureller Eigenschaften dargestellten Situation in einer Textaufgabe von den Lernenden spontan oder aufgrund eines einfachen Hinweises erkannt und für die Lösung einer folgenden Textaufgabe genutzt wird.

Dies könnte mehrere Ursachen haben:

1. Aufgrund der Ergebnisse zum ersten Fragenkomplex ist es durchaus möglich, dass Lernende eine solche Nutzung überhaupt nicht als hilfreich empfinden, da die beiden Aufgaben eines Textaufgaben-Paares nicht substantiell unterschiedlich schwierig sind. Dies würde dafür sprechen, in einer eventuellen Förderung auch auf die Flexibilität in Bezug auf andere sprachlich kodierte Informationen in Textaufgaben, wie z. B. die Richtung quantitativer Vergleiche und Veränderungen, einzugehen.

2. Es könnte sein, dass Lernende zwar zur Nutzung der strukturellen Ähnlichkeit in der Lage sind, dieses Wissen jedoch aufgrund soziomathematischer Normen dazu, wie eine Textaufgabe zu lösen ist, nicht nutzen (Yackel und Cobb 1996). Dass ein entsprechender expliziter Hinweis zur Nutzung der strukturellen Ähnlichkeit zwischen den Aufgaben keinen Effekt hatte, spricht gegen diese Erklärung.

3. Es könnte sein, dass Lernende diese Strategie aus anderen Gründen nicht anwenden, obwohl sie dazu in der Lage wären. Es wäre dann eine offene Frage, welche Gründe dies sein könnten, und ob ein entsprechendes Förderkonzept die Nutzung der Strategie anregen bzw. intensivieren kann.

4. Es könnte sein, dass Lernende im untersuchten Alter prinzipiell nicht in der Lage sind, die hier angenommene Umdeutungsstrategie zu erwerben. Uns sind keine Ergebnisse (z. B. aus der Entwicklungspsychologie) bekannt, die auf eine solche prinzipielle Einschränkung hinweisen. Im Gegenteil hat die entwicklungspsychologische Forschung in vielen Bereichen in der Vergangenheit sehr frühe Fähigkeiten bei eigentlich als ,abstrakt“ angenommenen Anforderungen dokumentiert, 
wenn diese in konkrete und zugängliche Kontexte (wie bei den hier betrachteten Textaufgaben) eingebettet sind (z. B. Markovits und Thompson (2008) für logisches Schließen; Koerber et al. (2015) für wissenschaftliches Denken). Letztlich lässt sich die Frage nach der Erlernbarkeit einer Strategie aber auch nicht mithilfe von Beobachtungsstudien, sondern lediglich anhand von Trainingsstudien beantworten.

5. Es ist zudem möglich, dass die Lernenden schlicht nicht über die notwendige Flexibilität im Umgang mit Situationsstrukturen verfügen, um die strukturelle Ähnlichkeit der Aufgaben aus den sprachlichen Beschreibungen zu entnehmen. Es wäre in diesem Fall eine wesentliche Frage, ob das angedachte Förderkonzept geeignet ist, eine solche Flexibilität anzuregen und diese für das Lösen von Textaufgaben nutzbar zu machen.

Um die letzten beiden Erklärungsansätze zu trennen, wäre es hilfreich, Flexibilität im Umgang mit Situationsstrukturen in Bezug auf additive Situationen unabhängig von Textaufgaben erheben zu können. Ein erstes Instrument dafür liegt aus einer Examensarbeit bereits vor (Weber 2016) und wurde erfolgreich pilotiert. Ergebnisse dieser Arbeit deuten darauf hin, dass ein Teil der Lernenden in der Lage ist, die strukturelle Ähnlichkeit unterschiedlich beschriebener additiver Situationen zu erkennen und beim Lösen von Textaufgaben zu nutzen. Dies schwächt insbesondere auch eventuelle prinzipielle Einwände gegen die Erlernbarkeit dieser Flexibilität.

Aufgrund der geringen Unterschiede in der Schwierigkeit von Textaufgaben zu unterschiedlichen semantischen Strukturen und der Ergebnisse zu Modellen des Zahlbegriffserwerbs (Fritz et al. 2018) scheint es plausibel, dass viele Lernende auch den Vergleichsaufgaben zugrundeliegenden anspruchsvollen Relationszahlaspekt bis zur der Mitte der zweiten Jahrgangsstufe im Kern beherrschen. Die Ergebnisse zu Fragenkomplex 1 deuten darauf hin, dass besonders eine Flexibilität in Bezug auf quantitative Vergleiche und Veränderungen an dieser Stelle der Entwicklung eine wesentliche Herausforderung darstellt. Dieser Entwicklungsschritt ist in bisherigen Modellen zur Zahlbegriffsentwicklung nicht systematisch beschrieben (z.B. Fritz et al. 2018). Sollte ein Förderkonzept, das diese Flexibilität anregt, Wirkungen auf die Fähigkeiten zum Lösen von Textaufgaben haben, so würde dies darauf hindeuten, dass das Verständnis dieser Symmetrie quantitativer Vergleiche sich erst zu diesem Zeitpunkt entwickelt. Offen wäre weiterhin die Frage, ob derartige Beziehungen gemeinsam für alle semantischen Strukturen erworben bzw. gefördert werden können, oder ob sie für bestimmte (z. B. dynamische) semantische Strukturen früher erworben werden als für andere (z.B. statische, auf den Relationszahlaspekt bezogene Strukturen).

\subsection{Limitationen der Studie}

Die vorliegende Studie kann aufgrund ihres Designs sicher auf viele Fragen keine finalen Antworten geben. Die herangezogene Stichprobe ist zwar geeignet, mittlere bis große Effekte von Aufgabenmerkmalen zu identifizieren, sie reicht jedoch sicher nicht aus, um kleine Unterschiede im Detail aufklären. Um die Konzeption eines Förderkonzepts zu fundieren, sind jedoch primär auch die Mechanismen von 
Interesse, die mit größeren Unterschieden in der Leistung der Lernenden einhergehen. Dennoch kann aus dieser Studie nicht geschlossen werden, dass einzelne der betrachteten Faktoren nicht doch eine gewisse Relevanz für das Lösen von Textaufgaben haben, die sich jedoch mit der gewählten Methodik nicht in Form signifikanter Effekte absichern ließen.

Entsprechend kann die Studie aufgrund ihres experimentellen Designs zwar Aussagen zu kausalen Zusammenhängen zwischen Aufgabenmerkmalen und der Aufgabenschwierigkeit machen, es bleiben jedoch Fragen offen. So kann diese Studie zwar wertvolle Informationen zur Konzeption eines Förderkonzepts liefern, jedoch sicher nichts über dessen potentielle Wirkung aussagen.

Auch die Auswahl der Kontexte und Situationstypen muss bei der Interpretation der Ergebnisse berücksichtigt werden. Ziel der Studie war es zu untersuchen, wie Lernende bestimmte Anforderungen beim Umgang mit einfachen, verbal beschriebenen additiven Situationen bewältigen. Das Spektrum der betrachteten Situationen wurde deshalb bewusst beschränkt. So wurden keine Vereinigungsaufgaben einbezogen, da diese sich strukturell weniger gut an die als besonders schwierig erachteten Vergleichsaufgaben anbinden lassen. Andererseits wurden nur einfache, eingekleidete Simplexaufgaben betrachtet. Wie Lernende die hier untersuchten Fähigkeiten und ihr Wissen über additive Situationen in komplexeren, ggf. auch authentischeren Sachsituationen anwenden, geht deutlich über den Fokus dieses Beitrags hinaus.

\subsection{Zusammenfassung und Implikationen}

Dieser Beitrag nimmt einen besonderen Blick auf die Rolle von Sprache beim Erlernen mathematischer Konzepte ein. Flexibilität im Umgang mit mathematischen Situationsstrukturen beim Erschließen von additiven Textaufgaben beschreibt die Fähigkeit von Lernenden, Situationsmodelle zu diesen Situationen durch Inferenzen mit zusätzlichen strukturellen Eigenschaften auf der Ebene der Situationsstrukturen (Abb. 1) anzureichern. Damit schlägt der Beitrag ein theoretisches Konzept vor, das zunächst weitgehend sprachunabhängig erscheint. Es ist jedoch von wesentlicher Bedeutung für das Verständnis von sprachlichen Informationen zu Situationen, die mathematisch beschrieben werden sollen: Gerade die konkreten sprachlichen Realisierungen solcher Situationen in Textaufgaben heben eben meist nur einen Ausschnitt der strukturellen Eigenschaften einer Situation hervor, während andere strukturelle Eigenschaften und alternative Sichtweisen durch zusätzliche Inferenzen angereichert werden müssen. Wie sich die dafür notwendige Flexibilität zu fachsprachlichen Konzepten wie z. B. dem Fachwortschatz (Schindler et al. 2019; Ufer und Bochnik 2020; Peng und Lin 2019; Powell et al. 2017) und Fähigkeiten zum textintegrativen Verständnis (Bochnik und Ufer 2016; Ufer und Bochnik 2020), zu domänenunabhängigen sprachlichen Kompetenzen oder zu weniger sprachbezogenen Teilen des Begriffsverständnisses verhält, wäre in weiteren Studien abzuklären. Eine weitere interessante Anschlussfrage wäre darüber hinaus, inwiefern eine solche zunehmende Flexibilität zum Aufbau einer vernetzten Wissensstruktur zu mathematischen additiven Situationen beiträgt, welche dann im Sinne eines ,,abstrakten MetaSchemas“ alle Situationstypen integriert. 
Über die bereits angesprochenen Folgerungen für das geplante Förderkonzept hinaus ergeben sich aus der Studie einige wesentliche Implikationen.

1. Die in der bisherigen, z. T. älteren Literatur in Jahrgangsstufe 1 (z. B. Stern 1998) beschriebenen Schwierigkeitsunterschiede zwischen verschiedenen semantischen Strukturen lassen sich zum jetzigen Zeitpunkt für unsere Stichprobe zur Mitte der Jahrgangsstufe 2 nicht vollständig replizieren. Auch wenn Ergebnisse zum Zahlbegriffserwerb nahelegen, dass etwa ein Viertel der Lernenden zu Beginn der zweiten Jahrgangsstufe ein Verständnis für Relationszahlen aufgebaut haben, ist es doch bemerkenswert, dass dieser bereits Mitte der Jahrgangsstufe 2 kein wirkliches Hindernis in den Aufgaben in unserer Studie mehr darzustellen scheint. Die initiale Annahme, dass eine Umdeutung verschiedener semantischer Strukturen besonders hilfreich sein könnte, wird in der Folge durch die Studie vorerst nicht gestützt.

2. Stattdessen rückt die Studie das Verständnis der Symmetrie quantitativer Vergleiche und Veränderungen in den Blick. Auch wenn es zunächst plausibel erscheint, dass diese Symmetrie besonders bei Vergleichsaufgaben relevant wird, weisen unsere Ergebnisse doch darauf hin, dass entsprechende Schwierigkeitsunterschiede bei den anderen beiden betrachteten semantischen Strukturen nicht signifikant schwächer ausgeprägt sind. Dies spricht dafür, dass sich ein Verständnis der Symmetrie quantitativer Vergleiche und Veränderungen erst nach dem Erwerb des Relationszahlbegriffs aufbaut. Es konnten keine Hinweise gefunden werden, dass dies für bestimmte semantische Strukturen einfacher oder früher geschieht als für andere. Insgesamt spricht dies dafür, bei der Bearbeitung sprachlicher Mittel zur Beschreibung von quantitativen Vergleichen alle semantischen Strukturen, und somit insbesondere auch Veränderungen, gleichermaßen in den Blick zu nehmen. In Bezug auf das angedachte Förderkonzept legt die Studie also nahe, die Symmetrie quantitativer Vergleiche und Veränderungen in Bezug auf alle semantischen Strukturen zu thematisieren, und in diesem Sinne einen flexiblen Umgang mit mathematischen Situationsstrukturen anzuregen.

3. Die Studie deutet darauf hin, dass Lernende mathematische Modelle auf Basis der Addition gegenüber Modellen auf Basis der Subtraktion bevorzugen. Hierfür sind unterschiedliche Ursachen denkbar, von individuellen Bewertungen der Operationen (z. B. Additionen als einfacher) bis hin zu Erklärungen bezüglich des Lernangebots. So wäre zu prüfen, inwiefern Sachsituationen zu Addition und Subtraktion gleich häufig und auch in direkter zeitlicher Kontingenz im Unterricht thematisiert werden. Eine systematische Bevorzugung bestimmter Operationen, unabhängig von der Aufgabenstellung, kann auf Probleme beim Verständnis von additiven Situationen hinweisen. Bei ungünstiger Aufgabenauswahl ist jedoch auch denkbar, dass für Lernende eine Verstehensillusion entsteht, wenn sie mit der bevorzugten Strategie sehr häufig zum Erfolg kommen.

4. Letztlich gibt die Studie keine Hinweise auf eine spontane Nutzung der Strukturähnlichkeit direkt aufeinander folgender Aufgaben. Es zeigen sich auch keine Hinweise, dass dies lediglich ein Effekt entsprechender sozio-mathematischer Normen im Unterrichtskontext wäre. Auch wenn diese spezifische Nutzung sicher kein zentrales Ziel des Mathematikunterrichts ist, weist es doch darauf hin, 
dass der Aufbau und die Nutzung flexiblen Wissens zu additiven Situationen nicht als gelöstes Problem der Mathematikdidaktik angesehen werden kann. Weitere Forschung sollte untersuchen, ob und unter welchen Bedingungen Lernende Ähnlichkeiten zwischen additiven Situationsstrukturen zum Lösen von Textaufgaben heranziehen können.

Um die Wirkmechanismen hinter den identifizierten und nicht identifizierten $\mathrm{Zu}$ sammenhängen besser zu verstehen, ist eine detaillierte Analyse von individuellen Problemlöse- und Lernprozessen notwendig, wie sie z.B. anhand einer Trainingsstudie erfolgen könnte. Denkbar wäre auch die Analyse kognitiver Prozesse im Rahmen von qualitativen Analysen. Mithilfe der Methode des Lauten Denkens könnten Gedankengänge und Vorgehensweisen der Lernenden beim Bearbeiten der Textaufgaben-Paare ergründet werden.

Zusammenfassend prüft und systematisiert die vorliegende Studie ältere Ergebnisse zu Textaufgaben mit additiver Struktur. Dabei zeigt sich ein durchaus differenzierteres Bild als im bisherigen Forschungsstand verankert. Bezüglich des geplanten Förderkonzepts kann die Studie wesentliche Informationen zur Ausrichtung der Förderziele beitragen. Die Annahme, dass die im Förderkonzept aufzubauende Flexibilität im Umgang mit mathematischen Situationsstrukturen bereits in deutlichem Maße genutzt wird, konnte nicht bestätigt werden. Die Auswirkungen einer Förderung dieser Flexibilität können lediglich in einer Interventionsstudie untersucht werden. Über das Förderkonzept hinaus liefert die Studie Hinweise darauf, dass ein - theoretisch durchaus wünschenswerter - flexibler Umgang mit Situationsstrukturen nicht systematisch als Strategie genutzt wird, um sprachliche Anforderungen in Textaufgaben zu bewältigen. Dies spricht dafür, mögliche Hürden für die Anwendung dieser Strategie zu beforschen.

Funding Open Access funding provided by Projekt DEAL.

Open Access Dieser Artikel wird unter der Creative Commons Namensnennung 4.0 International Lizenz veröffentlicht, welche die Nutzung, Vervielfältigung, Bearbeitung, Verbreitung und Wiedergabe in jeglichem Medium und Format erlaubt, sofern Sie den/die ursprünglichen Autor(en) und die Quelle ordnungsgemäß nennen, einen Link zur Creative Commons Lizenz beifügen und angeben, ob Änderungen vorgenommen wurden.

Die in diesem Artikel enthaltenen Bilder und sonstiges Drittmaterial unterliegen ebenfalls der genannten Creative Commons Lizenz, sofern sich aus der Abbildungslegende nichts anderes ergibt. Sofern das betreffende Material nicht unter der genannten Creative Commons Lizenz steht und die betreffende Handlung nicht nach gesetzlichen Vorschriften erlaubt ist, ist für die oben aufgeführten Weiterverwendungen des Materials die Einwilligung des jeweiligen Rechteinhabers einzuholen.

Weitere Details zur Lizenz entnehmen Sie bitte der Lizenzinformation auf http://creativecommons.org/ licenses/by/4.0/deed.de. 


\section{Anhang}

Tab. 2 Beispiele für Situationstypen von additiven Simplex-Textaufgaben unter Berücksichtigung der Formulierungsrichtung (angelehnt an Stern 1998); genannt sind jeweils die in Abb. 1 genutzten gemeinsamen Bezeichnungen der Mengen und die in der Literatur verwendeten spezifischen Bezeichnungen je nach semantischer Struktur

\begin{tabular}{|c|c|}
\hline $\begin{array}{l}\text { Semantische Struktur/ } \\
\text { Gesuchte Menge }\end{array}$ & Beispiel \\
\hline \multicolumn{2}{|l|}{ Vereinigung } \\
\hline $\begin{array}{l}\text { Teilmenge }(\mathrm{T}) \\
\text { unbekannt }\end{array}$ & $\begin{array}{l}\text { Anna und Ben haben zusammen } 15 \text { Murmeln. Anna hat } 8 \text { Murmeln. Wie } \\
\text { viele Murmeln hat Ben? }\end{array}$ \\
\hline $\begin{array}{l}\text { Gesamtmenge }(\mathrm{G}) \\
\text { unbekannt }\end{array}$ & $\begin{array}{l}\text { Anna hat } 8 \text { Murmeln. Ben hat } 7 \text { Murmeln. Wie viele Murmeln haben sie } \\
\text { zusammen? }\end{array}$ \\
\hline \multicolumn{2}{|l|}{ Veränderung } \\
\hline \multirow[t]{2}{*}{$\begin{array}{l}\text { Ausgangsmenge }(\mathrm{A}) \\
\text { unbekannt }\end{array}$} & $\begin{array}{l}\text { Am Anfang hatte Anna einige Murmeln. Dann hat sie } 7 \text { Murmeln dazu- } \\
\text { bekommen. Jetzt hat Anna } 15 \text { Murmeln. Wie viele Murmeln hatte sie am } \\
\text { Anfang? }\end{array}$ \\
\hline & $\begin{array}{l}\text { Am Anfang hatte Anna einige Murmeln. Dann hat sie } 7 \text { Murmeln abgegeben. } \\
\text { Jetzt hat Anna } 8 \text { Murmeln. Wie viele Murmeln hatte Anna am Anfang? }\end{array}$ \\
\hline \multirow[t]{2}{*}{$\begin{array}{l}\text { Veränderungsmenge (V) } \\
\text { unbekannt }\end{array}$} & $\begin{array}{l}\text { Anna hatte } 8 \text { Murmeln. Dann hat sie noch einige dazubekommen. Jetzt hat } \\
\text { Anna } 15 \text { Murmeln. Wie viele Murmeln hat Anna dazubekommen? }\end{array}$ \\
\hline & $\begin{array}{l}\text { Anna hatte } 15 \text { Murmeln. Dann hat sie einige davon abgegeben. Jetzt hat } \\
\text { Anna } 8 \text { Murmeln. Wie viele Murmeln hat Anna abgegeben? }\end{array}$ \\
\hline \multirow[t]{2}{*}{$\begin{array}{l}\text { Ergebnismenge }(\mathrm{E}) \\
\text { unbekannt }\end{array}$} & $\begin{array}{l}\text { Anna hatte } 8 \text { Murmeln. Dann hat sie } 7 \text { Murmeln dazubekommen. Wie viele } \\
\text { Murmeln hat Anna jetzt? }\end{array}$ \\
\hline & $\begin{array}{l}\text { Anna hatte } 15 \text { Murmeln. Dann hat sie } 7 \text { davon abgegeben. Wie viele Mur- } \\
\text { meln hat Anna jetzt? }\end{array}$ \\
\hline \multicolumn{2}{|l|}{ Vergleich } \\
\hline \multirow{2}{*}{$\begin{array}{l}\text { Ausgangsmenge (A) } \\
\text { bzw. Referenzmenge } \\
\text { unbekannt }\end{array}$} & $\begin{array}{l}\text { Anna hat } 15 \text { Murmeln. Anna hat } 7 \text { Murmeln mehr als Ben. Wie viele Mur- } \\
\text { meln hat Ben? }\end{array}$ \\
\hline & $\begin{array}{l}\text { Anna hat } 8 \text { Murmeln. Anna hat } 7 \text { Murmeln weniger als Ben. Wie viele Mur- } \\
\text { meln hat Ben? }\end{array}$ \\
\hline \multirow{2}{*}{$\begin{array}{l}\text { Veränderungsmenge }(\mathrm{V}) \\
\text { bzw. Differenzmenge } \\
\text { unbekannt }\end{array}$} & $\begin{array}{l}\text { Anna hat } 15 \text { Murmeln, Ben hat } 8 \text { Murmeln. Wie viele Murmeln hat Anna } \\
\text { mehr als Ben? }\end{array}$ \\
\hline & $\begin{array}{l}\text { Anna hat } 15 \text { Murmeln, Ben hat } 8 \text { Murmeln. Wie viele Murmeln hat Ben } \\
\text { weniger als Anna? }\end{array}$ \\
\hline \multirow{2}{*}{$\begin{array}{l}\text { Ergebnismenge }(\mathrm{E}) \\
\text { bzw. Vergleichsmenge } \\
\text { unbekannt }\end{array}$} & $\begin{array}{l}\text { Anna hat } 8 \text { Murmeln. Ben hat } 7 \text { Murmeln mehr als Anna. Wie viele Mur- } \\
\text { meln hat Ben? }\end{array}$ \\
\hline & $\begin{array}{l}\text { Anna hat } 15 \text { Murmeln. Ben hat } 7 \text { Murmeln weniger als Anna. Wie viele } \\
\text { Murmeln hat Ben? }\end{array}$ \\
\hline
\end{tabular}


Tab. 2 (Fortsetzung)

Semantische Struktur/ Beispiel
Gesuchte Menge

Ausgleich

Ausgangsmenge (A)

unbekannt

Anna hat 15 Murmeln. Ben muss noch 7 Murmeln bekommen, damit er gleich viele wie Anna hat. Wie viele Murmeln hat Ben?

Anna hat 8 Murmeln. Ben muss noch 7 Murmeln abgeben, damit er gleich viele wie Anna hat. Wie viele Murmeln hat Ben?

Veränderungsmenge (V) Anna hat 15 Murmeln, Ben hat 8 Murmeln. Wie viele Murmeln muss Ben unbekannt noch bekommen, damit er gleich viele wie Anna hat?

Anna hat 15 Murmeln, Ben hat 8 Murmeln. Wie viele Murmeln muss Anna noch abgeben, damit sie gleich viele wie Ben hat?

Ergebnismenge (E) Anna hat 8 Murmeln. Sie muss noch 7 Murmeln bekommen, damit sie gleich unbekannt viele wie Ben hat. Wie viele Murmeln hat Ben?

Anna hat 15 Murmeln. Sie muss noch 7 Murmeln abgeben, damit sie gleich viele wie Ben hat. Wie viele Murmeln hat Ben?

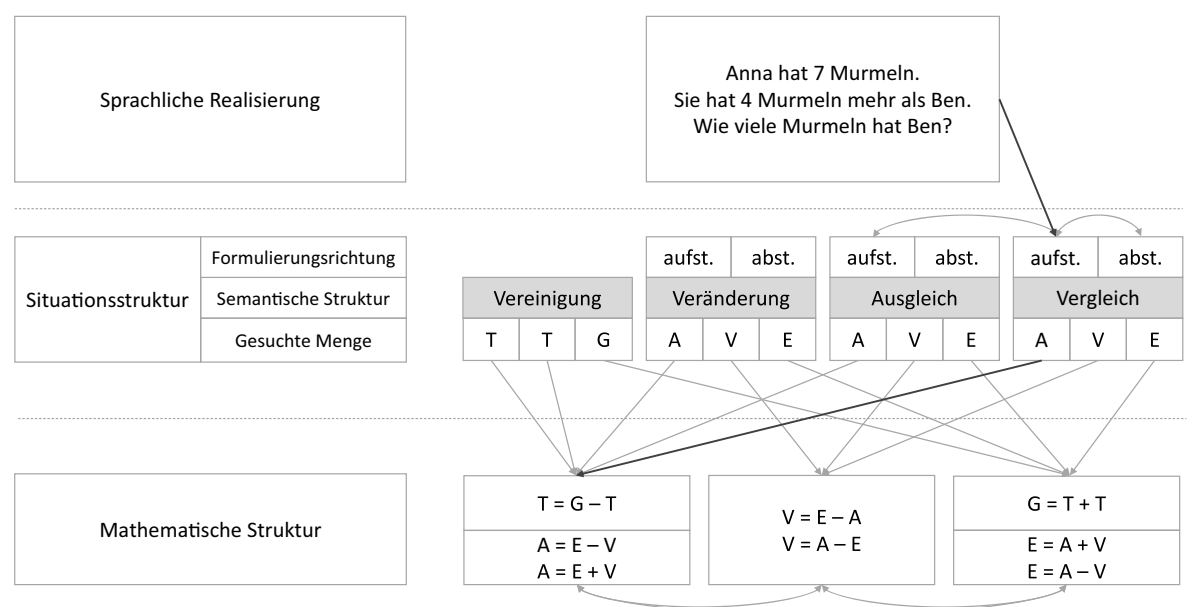

Abb. 3 Vom Autor intendierte Struktur einer exemplarischen additiven Textaufgabe (hervorgehoben werden die Situationsstruktur und die mathematische Struktur, die vom Autor zur Darstellung der Situation intendiert wurden) 


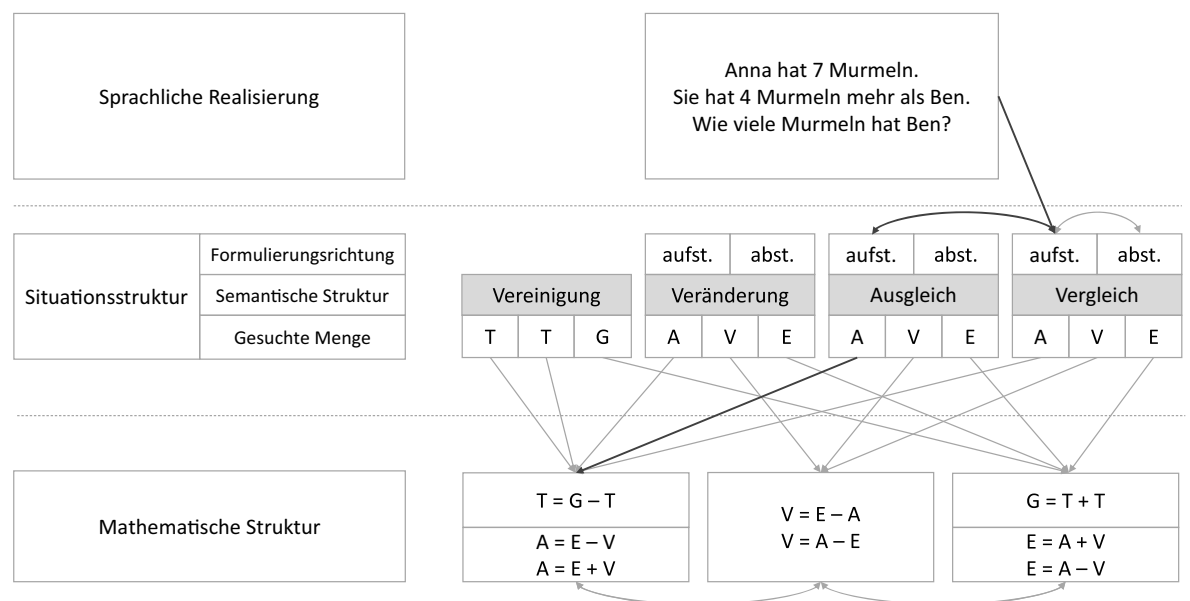

Abb. 4 Von einem Lerner potentiell rekonstruierte Strukturen beim Lösen einer exemplarischen additiven Textaufgabe (hervorgehoben ist die Rekonstruktion der vom Autor intendierten Situationsstruktur, deren Umdeutung in eine alternative Situationsstruktur und deren Umsetzung in eine mathematische Struktur)

\section{Literatur}

Abedi, J., Courtney, M., Leon, S., Kao, J., \& Azzam, T. (2006). English language learners and math achievement: a study of opportunity to learn and language accomodation. Los Angeles: National Center for Research on Evaluation, Standards, and Student Testing, University of California.

Bailey, A. L., \& Butler, F. A. (2003). An evidentiary framework for operationalizing academic language for broad application to K-12 education: a design document. Los Angeles: National Center for Research on Evaluation, Standards, and Student Testing, University of California.

Bates, D., Maechler, M., Bolker, B., \& Walker, S. (2014). lme4: Linear mixed-effects models using Eigen and S4. R package version, 1(7), 1-23.

Baumert, J., \& Schümer, G. (2001). Familiäre Lebensverhältnisse, Bildungsbeteiligung und Kompetenzerwerb. In J. Baumert, E. Klieme, M. Neubrand, M. Prenzel, U. Schiefele \& W. Schneider, et al. (Hrsg.), PISA 2000. Basiskompetenzen von Schülerinnen und Schülern im internationalen Vergleich (S. 323-410). Opladen: Leske + Budrich.

Benz, C. (2005). Erfolgsquoten, Rechenmethoden, Lösungswege und Fehler von Schülerinnen und Schülern bei Aufgaben zur Addition und Subtraktion im Zahlenraum bis 100. Hildesheim: Franzbecker.

Blum, W., \& Leiß, D. (2005). Modellieren im Unterricht mit der „Tanken“-Aufgabe. mathematik lehren, $128,18-46$.

Bochnik, K. (2017). Sprachbezogene Merkmale als Erklärung für Disparitäten mathematischer Leistung: Differenzierte Analysen im Rahmen einer Längsschnittstudie in der dritten Jahrgangsstufe. Münster: Waxmann.

Bochnik, K., \& Ufer, S. (2016). Die Rolle (fach-)sprachlicher Kompetenzen zur Erklärung mathematischer Kompetenzunterschiede zwischen Kindern mit deutscher und nicht-deutscher Familiensprache. Zeitschrift für Grundschulforschung, 9(1), 135-147.

Breidenbach, W. (1969). Methodik des Mathematikunterrichts in Grund- und Hauptschulen. Hannover: Schroedel.

Carpenter, T.P., Hiebert, J., \& Moser, J.M. (1981). Problem structure and first-grade children's initial solution processes for simple addition and subtraction problems. Journal for Research in Mathematics Education, 12(1), 27-39.

Civil, M. (2008). Language and mathematics: Immigrant parents' participation in school. In O. Figueras, J. L. Cortina, S. Alatorre, T. Rojano \& A. Sepúlveda (Hrsg.), Proceedings of the Joint Meeting of the 32nd Conference of the International Group for the Psychology of Mathematics Education and the XX North American Chapter (Bd. 2, S. 329-336). Morelia: PME. 
Cummins, D.D., Kintsch, W., Reusser, K., \& Weimer, R. (1988). The role of understanding in solving word problems. Cognitive Psychology, 20(4), 405-438.

Franke, M., \& Ruwisch, S. (2010). Didaktik des Sachrechnens in der Grundschule. Mathematik Primarstufe und Sekundarstufe I + II. Heidelberg: Spektrum.

Fritz, A., Ehlert, A., \& Leutner, D. (2018). Arithmetische Konzepte aus kognitiv-entwicklungspsychologischer Sicht. Journal für Mathematik-Didaktik, 39(1), 7-41.

Fromme, M., Wartha, S., \& Benz, C. (2011). Grundvorstellungen zur Subtraktion: Tragfähiges Operationsverständnis durch flexible Übersetzungen. Grundschulmagazin, 4, 35-40.

Fuson, K.C., Carroll, W.M., \& Landis, J. (1996). Levels in conceptualizing and solving addition and subtraction compare word problems. Cognition and Instruction, 14(3), 345-371.

Greefrath, G., Kaiser, G., Blum, W., \& Borromeo Ferri, R. (2013). Mathematisches Modellieren: Eine Einführung in theoretische und didaktische Hintergründe. In R. Borromeo Ferri, G. Greefrath \& G. Kaiser (Hrsg.), Mathematisches Modellieren für Schule und Hochschule (S. 11-37). Wiesbaden: Springer.

Greeno, J. G. (1980). Some examples of cognitive task analysis with instructional implications. In E. Snow, P.-A. Frederico \& W.E. Montague (Hrsg.), Cognitive process analysis of learning and problem solving. Aptitude, learning, and instruction, (Bd. 2, S. 1-21). Hillsdale, NJ: Lawrence Erlbaum.

Haag, N., Heppt, B., Stanat, P., Kuhl, P., \& Pant, H. A. (2013). Second language learners' performance in mathematics: disentangling the effects of academic language features. Learning and Instruction, 28, 24-34.

Heinze, A., Herwartz-Emden, L., \& Reiss, K. (2007). Mathematikkenntnisse und sprachliche Kompetenz bei Kindern mit Migrationshintergrund zu Beginn der Grundschulzeit. Zeitschrift für Pädagogik, 53(4), 562-581.

vom Hofe, R. (1995). Grundvorstellungen mathematischer Inhalte. Heidelberg: Spektrum.

Kempert, S., Schalk, L., \& Saalbach, H. (2018). Übersichtsartikel: Sprache als Werkzeug des Lernens: Ein Überblick zu den kommunikativen und kognitiven Funktionen der Sprache und deren Bedeutung für den fachlichen Wissenserwerb. Psychologie in Erziehung und Unterricht, 56, 1-20.

Kintsch, W. (1998). Comprehension: a paradigm for cognition. Cambridge: Cambridge University Press.

Kintsch, W., \& Greeno, J. G. (1985). Understanding and solving word arithmetic problems. Psychological Review, 92(1), 109-129.

Klieme, E. (2004). Was sind Kompetenzen und wie lassen sie sich messen? Pädagogik, 56(6), 10-13.

Klieme, E., Avenarius, H., Blum, W., Döbrich, P., Gruber, H., Prenzel, M., et al. (2003). Zur Entwicklung nationaler Bildungsstandards: Eine Expertise. Berlin: BMBF.

Koerber, S., Mayer, D., Osterhaus, C., Schwippert, K., \& Sodian, B. (2015). The development of scientific thinking in elementary school: a comprehensive inventory. Child Development, 86(1), 327-336.

Krajewski, K., \& Schneider, W. (2009). Early development of quantity to number-word linkage as a precursor of mathematical school achievement and mathematical difficulties: findings from a four-year longitudinal study. Learning and Instruction, 19(6), 513-526.

Lenth, H., Singmann, H., Love, J., Buerkner, P., \& Herve, M. (2018). Package 'emmeans': R package version 1.3.1. http://CRAN.R-project.org/package=emmeans. Zugriff am 18.05.2020

Markovits, H., \& Thompson, V. (2008). Different developmental patterns of simple deductive and probabilistic inferential reasoning. Memory \& Cognition, 36(6), 1066-1078.

Modestou, M., \& Gagatsis, A. (2013). A didactical situation for the enhancement of meta-analogical awareness. Journal of Mathematical Behavior, 32(2), 160-172.

Moschkovich, J. (2007). Using two languages when learning mathematics. Educational Studies in Mathematics, 64(2), 121-144.

Nesher, P., Greeno, J.G., \& Riley, M.S. (1982). The development of semantic categories for addition and subtraction. Educational Studies in Mathematics, 13(4), 373-394.

Paetsch, J. (2016). Der Zusammenhang zwischen sprachlichen und mathematischen Kompetenzen bei Kindern deutscher und bei Kindern nicht-deutscher Familiensprache. Berlin: Freie Universität Berlin.

Paetsch, J., Radmann, S., Felbrich, A., Lehmann, R., \& Stanat, P. (2016). Sprachkompetenz als Prädiktor mathematischer Kompetenzentwicklung von Kindern deutscher und nicht-deutscher Familiensprache. Zeitschrift für Entwicklungspsychologie und Pädagogische Psychologie, 48, 27-41.

Peng, P., \& Lin, X. (2019). The relation between mathematics vocabulary and mathematics performance among fourth graders. Learning and Individual Differences, 69, 11-21.

Powell, S. R., Driver, M. K., Roberts, G., \& Fall, A.-M. (2017). An analysis of the mathematics vocabulary knowledge of third-and fifth-grade students: Connections to general vocabulary and mathematics computation. Learning and Individual Differences, 57, 22-32. 
Prediger, S., \& Wessel, L. (2013). Fostering German-language learners' constructions of meanings for fractions: design and effects of a language- and mathematics-integrated intervention. Mathematics Education Research Journal, 25(3), 435-456.

Radatz, H. (1983). Untersuchungen zum Lösen eingekleideter Aufgaben. Journal für Mathematik-Didaktik, 4(3), 205-217.

Radatz, H., Schipper, W., Ebeling, A., \& Dröge, R. (1996). Handbuch für den Mathematikunterricht. Hannover: Schroedel.

Resnick, L. B., \& Singer, J.A. (1993). Protoquantitative origins of ratio reasoning. In T.P. Carpenter, E. Fennema \& T. A. Romberg (Hrsg.), Rational numbers: an integration of research (S. 107-130). Hillsdale: Lawrence Erlbaum.

Riley, M.S., \& Greeno, J.G. (1988). Developmental analysis of understanding language about quantities and of solving problems. Cognition and Instruction, 5(1), 49-101.

Riley, M.S., Greeno, J.G., \& Heller, J.I. (1983). Development of children's problem-solving ability in arithmetic. In H.P. Ginsburg (Hrsg.), The development of mathematical thinking (S. 153-196). New York: Academic Press.

Schindler, V., Moser-Opitz, E., Cadonau-Bieler, M., \& Ritterfeld, U. (2019). Überprüfung und Förderung des mathematischen Fachwortschatzes der Grundschulmathematik: Eine empirische Studie. Journal für Mathematik-Didaktik, 40(1), 1-35.

Schipper, W. (2009). Handbuch für den Mathematikunterricht an Grundschulen. Braunschweig: Schroedel.

Schmidt, S. (2009). Arithmetische Kenntnisse am Schulanfang. In A. Fritz, G. Ricken \& S. Schmidt (Hrsg.), Handbuch Rechenschwäche: Lernwege, Schwierigkeiten und Hilfen bei Dyskalkulie (S. 77-99). Weinheim: Beltz.

Schütte, M. (2009). Sprache und Interaktion im Mathematikunterricht der Grundschule: Zur Problematik einer Impliziten Pädagogik für schulisches Lernen im Kontext sprachlich-kultureller Pluralität. Münster: Waxmann.

Sfard, A. (2008). Thinking as communicating: human development, the growth of discourses, and mathematizing. TMME, 5, 429-436.

Steenpaß, A., \& Steinbring, H. (2014). Young students' subjective interpretations of mathematical diagrams: elements of the theoretical construct "frame-based interpreting competence". ZDM Mathematics Education, 46(1), 3-14.

Stern, E. (1992). Warum werden Kapitänsaufgaben „gelöst“? Das Verstehen von Textaufgaben aus psychologischer Sicht. Der Mathematikunterricht, 38(5), 7-29.

Stern, E. (1993). What makes certain arithmetic word problems involving the comparison of sets so difficult for children? Journal of Educational Psychology, 85(1), 7-23.

Stern, E. (1994). Die Erweiterung des mathematischen Verständnisses mit Hilfe von Textaufgaben. Grundschule, 26(3), 23-25.

Stern, E. (1998). Die Entwicklung des mathematischen Verständnisses im Kindesalter. Lengerich: Pabst.

Torbeyns, J., De Smedt, B., Stassens, N., Ghesquière, P., \& Verschaffel, L. (2009). Solving subtraction problems by means of indirect addition. Mathematical Thinking and Learning, 11(1), 79-91.

Ufer, S., \& Bochnik, K. (2020). The role of general and subject-specific language skills when learning mathematics in elementary school. Journal für Mathematik-Didaktik. https://doi.org/10.1007/s13138020-00160-5.

Ufer, S., Reiss, K., \& Mehringer, V. (2013). Sprachstand, soziale Herkunft und Bilingualität: Effekte auf Facetten mathematischer Kompetenz. In M. Becker-Mrotzek, K. Schramm, E. Thürmann \& H. J. Vollmer (Hrsg.), Sprache im Fach (S. 185-202). Münster: Waxmann.

Van Dooren, W., De Bock, D., Vleugels, K., \& Verschaffel, L. (2010). Just answering... or thinking? Contrasting pupils' solutions and classifications of missing-value word problems. Mathematical Thinking and Learning, 12(1), 20-35.

Verschaffel, L., De Corte, E., \& Lasure, S. (1994). Realistic considerations in mathematical modeling of school arithmetic word problems. Learning and Instruction, 4(4), 273-294.

Verschaffel, L., Greer, B., \& De Corte, E. (2000). Making sense of word problems. Lisse: Swets \& Zeitlinger.

Verschaffel, L., Schukajlow, S., Star, J., \& Van Dooren, W. (2020). Word problems in mathematics education: a survey. ZDM Mathematics Education, 52, 1-16. https://doi.org/10.1007/s11858-020-011304.

Vicente, S., Orrantia, J., \& Verschaffel, L. (2008). Influence of situational and mathematical information on situationally difficult word problems. Studia Psychologica, 50(4), 337-356.

Vollrath, H.-J. (1989). Funktionales Denken. Journal für Mathematik-Didaktik, 10, 3-37. 
Weber, C. (2016). Analyse des flexiblen Umgangs mit situationsspezifischen Grundvorstellungen zu Addition und Subtraktion in Sachkontexten unter Berücksichtigung von Familiensprache, Sprachstand und textaufgabenbezogener Mathematikkompetenz. Schriftliche Hausarbeit im 1. Staatsexamen für das Lehramt an Grundschulen. München: Ludwig-Maximilians-Universität. Eine quantitative Erhebung zum mathematischen Situationsverständnis von Drittklässlern

Wendt, H., Schwippert, K., \& Stubbe, T.C. (2016). Mathematische und naturwissenschaftliche Kompetenzen von Schülerinnen und Schülern mit Migrationshintergrund. In H. Wendt, W. Bos, C. Selter, O. Köller, K. Schwippert \& D. Kasper (Hrsg.), TIMSS 2015. Mathematische und naturwissenschaftliche Kompetenzen von Grundschulkindern in Deutschland im internationalen Vergleich (S. 317-331). Münster: Waxmann.

Yackel, E., \& Cobb, P. (1996). Sociomathematical norms, argumentation, and autonomy in mathematics. Journal for Research in Mathematics Education, 27(4), 458-477. 